\title{
Loosely bound three-body nuclear systems in the $J$-matrix approach
}

\author{
Yu. A. Lurie $^{a}$ and A. M. Shirokov ${ }^{b}$ \\ ${ }^{a}$ The College of Judea and Samaria, Ariel 44837, Israel \\ ${ }^{\mathrm{b}}$ Skobeltsyn Institute of Nuclear Physics, Moscow State University, Moscow, \\ 119992, Russia
}

\begin{abstract}
We discuss the extension of the oscillator-basis $J$-matrix formalism on the case of true $A$-body scattering. The formalism is applied to loosely-bound ${ }^{11} \mathrm{Li}$ and ${ }^{6} \mathrm{He}$ nuclei within three-body cluster models ${ }^{9} \mathrm{Li}+n+n$ and $\alpha+n+n$. The $J$-matrix formalism is used not only for the calculation of the three-body continuum spectrum wave functions but also for the calculation of the $S$-matrix poles associated with the ${ }^{11} \mathrm{Li}$ and ${ }^{6} \mathrm{He}$ ground states to improve the description of the binding energies and ground state properties. The effect of the phase equivalent transformation of the $n-\alpha$ interaction on the properties of the ${ }^{6} \mathrm{He}$ nucleus is examined.
\end{abstract}

\section{Introduction}

In this paper we discuss a unified $J$-matrix approach to many-body systems. The approach links the quantum scattering theory formalism with traditional variational methods of nuclear theory based on the wave function expansion in the harmonic oscillator function series. The formalism extends the variational methods on the case of the continuum spectrum states. On the other hand, the variational description of the discrete spectrum states is improved by the use of the methods of the scattering theory. The $J$-matrix approach is supposed to be a valuable extension of the shell model and other nuclear structure models. Below we examine in detail the application of the $J$-matrix approach to the light weakly bound nuclei ${ }^{11} \mathrm{Li}$ and ${ }^{6} \mathrm{He}$ within the three-body cluster model.

Email addresses: ylurie@yosh.ac.il (Yu. A. Lurie), shirokov@nucl-th.sinp.msu.ru (A. M. Shirokov).

Preprint submitted to Elsevier Science 31 October 2018 
Weakly bound nuclei are extensively studied now both theoretically and experimentally. New methods are developed in the nuclear structure theory providing accurate description of the charge and matter distribution in the nuclear halo, allowing for the coupling to the continuum spectrum states, etc. At the same time, the traditional language of nuclear theory, i. e. the nuclear shell model, is based on the oscillator basis expansion of the wave functions and seems to be inadequate for the the continuum or weakly bound states with the wave functions slowly decreasing at large distances since the oscillator functions are rapidly decreasing asymptotically. We however believe that the traditional oscillator-basis nuclear theory can be successfully developed to face the above challenges by implementing the $J$-matrix formalism.

Within the $J$-matrix formalism, the continuum spectrum wave function is expanded in infinite series of $L^{2}$ functions. The $J$-matrix method was initially proposed in atomic physics $[1,2]$ and shown to be one of the most efficient and precise methods in calculations of photoionization $[3,4,5]$ and electron scattering by atoms [6]. In nuclear physics the same approach has been developed independently $[7,8]$ as the method of harmonic oscillator representation of scattering theory. This method has been successfully used in various nuclear applications allowing for two-body continuum channels, e. g. nucleus-nucleus scattering has been studied in the algebraic version of RGM based on the $J$ matrix formalism (see the review papers $[9,10]$ ); the effect of $\Lambda$ and neutron decay channels in hypernuclei production reactions has been investigated in Refs. [11,12], etc.

In this paper we study exotic neutron-excess nuclei ${ }^{11} \mathrm{Li}$ and ${ }^{6} \mathrm{He}$ in the threebody cluster models ${ }^{11} \mathrm{Li}={ }^{9} \mathrm{Li}+n+n$ and ${ }^{6} \mathrm{He}=\alpha+n+n$. The two-neutron separation energy in both of these nuclei is small compared with the neutron separation energy in the ${ }^{9} \mathrm{Li}$ and ${ }^{4} \mathrm{He}$ clusters; as a result the wave function of the pair of neutrons decreases slowly with distance and the rms radius of the two-neutron distribution is large compared with the rms radius of the ${ }^{9} \mathrm{Li}$ or ${ }^{4} \mathrm{He}$ core (the so-called two-neutron halo). We note that ${ }^{11} \mathrm{Li}$ and ${ }^{6} \mathrm{He}$ are the so-called Borromean nuclei, i. e. none of the two-body subsystems in the cluster decompositions ${ }^{11} \mathrm{Li}={ }^{9} \mathrm{Li}+n+n$ and ${ }^{6} \mathrm{He}=\alpha+n+n$ has a bound state. We employ the three-body extension of the $J$-matrix formalism for the description of the three-body decay channels and develop this approach to improve the description of the ground state properties.

Three-body decay channels are much more informative than the two-body ones, however the complete description of a three-body decay is a very complicated problem. Nevertheless in some cases, one can use the so-called democratic decay approximation for the successful analysis of the experimental data or for obtaining reliable theoretical predictions.

Generally, a three-body continuum spectrum wave function in the asymptotic 
region is a superposition of the components describing two- and three-body decay channels [13]. The democratic decay approximation accounts for the three-body channel only; two-body channels associated with the appearance of bound two-body subsystems and 'non-democratic' subdivision of the system of the type $(2+1)$, are not allowed for in the approximation. Therefore, this approximation is valid for the study of a three-body system only in the case when all two-body decay channels are closed and the only open channel is a three-body one; in other words, this is the case when none of the two-body subsystems has a bound state. Hence the democratic decay approximation is adequate for the study of Borromean nuclei ${ }^{6} \mathrm{He}$ and ${ }^{11} \mathrm{Li}$ at least at small enough excitation energies (less than the single nucleon binding energy in the ${ }^{9} \mathrm{Li}$ and ${ }^{4} \mathrm{He}$ clusters).

Generally speaking, if the three-body channel is the only open one, than the system can have two types of asymptotics [13]. One of the asymptotics corresponds to the situation when one of the particles is scattered by another and the third particle is a spectator. From the general physical point of view, this asymptotics is supposed to be of little importance for a nuclear system excited in some nuclear reaction and decaying via a three-body channel. The alternative type of the asymptotics is a superposition of ingoing and outgoing six-dimensional spherical waves, it corresponds to the situation when the decaying system emits (or/and absorbs) three particles from some point in space. Allowing for the three-body asymptotics of this type only is a quintessence of the democratic decay approximation; sometimes this approximation is also referred to as true three-body scattering or $3 \rightarrow 3$ scattering.

Nuclear structure studies in the framework of the democratic decay approximation have been started in 1970th by R. I. Jibuti and collaborators. The review of the results obtained by the Tbilisi group can be found in Refs. [14,15]. Recently a considerable progress has been made in both theoretical and experimental studies of democratic decays (see reviews in Refs. $[16,17,18,19]$ ).

The generalization of the $J$-matrix formalism on the case of $3 \rightarrow 3$ scattering (and on a more general case of $N \rightarrow N$ scattering) was suggested in Ref. [20]. First it was successfully applied in the study of monopole excitations in ${ }^{12} \mathrm{C}$ nucleus in the cluster model ${ }^{12} \mathrm{C}=\alpha+\alpha+\alpha$ in Ref. [21]. Later by means of this approach we studied $\Lambda \Lambda$ hypernuclei [22]. Our first attempts to employ this approach in the studies of ${ }^{11} \mathrm{Li}$ and ${ }^{6} \mathrm{He}$ nuclei in the three-body cluster models can be found in Refs. [23,24,25,26].

We use the $J$-matrix $3 \rightarrow 3$ scattering formalism for the construction of the three-body continuum spectrum wave functions of ${ }^{11} \mathrm{Li}$ and ${ }^{6} \mathrm{He}$ nuclei excited in nuclear reactions. However, probably more interesting is the application of this formalism to the study of the ground states of these nuclei treated as three-body systems ${ }^{9} \mathrm{Li}+n+n$ and $\alpha+n+n$. The idea is the following. 
By the $J$-matrix formalism we calculate the $3 \rightarrow 3$ scattering $S$-matrix. This calculation may be extended on the complex momentum plane. Hence we can locate numerically the $S$-matrix poles associated with the bound states. The $S$-matrix pole calculations improve the variational results for binding energies obtained by the pure diagonalization of the truncated Hamiltonian matrix. Knowing the binding energies, we calculate the bound state wave functions by means of the $J$-matrix formalism. We suppose that the approach based on the $S$-matrix pole calculation will be useful not only within the cluster models; the implementation of the $A \rightarrow A$ scattering $S$-matrix calculation within the $J$-matrix formalism is believed to improve essentially the traditional nuclear shell model.

The first calculation of the $S$-matrix poles within the $J$-matrix formalism was performed in Ref. [27] where resonances in $\alpha-\alpha$ scattering were investigated. The $S$-matrix poles in He atom and $\mathrm{H}^{-}$ion were calculated in Ref. [4,5] in the $J$-matrix model suggested by Broad and Reinhardt [3]. The resonance energies and widths were reproduced with a high accuracy. The $S$-matrix poles associated with the bound states were also calculated in Ref. [4,5]. The binding energies were reproduced with a very high accuracy; we note also that a very large number of bound states was obtained by means of the $S$-matrix pole calculations: it was not only much larger than the number of bound states obtained variationally (by the diagonalization of the truncated Hamiltonian matrix), but even much larger than the rank of the truncated Hamiltonian matrix. This approach not only provides an essential improvement in calculations of binding energies, but also makes it possible to calculate the ground state wave function with the correct asymptotics. It is very important for halo nuclei like ${ }^{11} \mathrm{Li}$ and ${ }^{6} \mathrm{He}$ due to the slow decrease of the wave function in the asymptotic region that cannot be reproduced by a superposition of a finite number of oscillator functions obtained in variational calculation. As a result, the rms radius, electromagnetic transition probabilities and other observables are improved essentially.

We also discuss a phase-equivalent transformation suggested in Ref. [26] that is used to obtain families of phase-equivalent $n-\alpha$ potentials. The effect of the phase-equivalent transformation of the $n-\alpha$ interaction on the ${ }^{6} \mathrm{He}$ properties is examined.

We start the discussion with a brief sketch of the $N \rightarrow N$ scattering $J$-matrix formalism suggested in Ref. [20]. 


\section{$2 J$-matrix formalism with hyperspherical oscillator basis}

The wave function $\Psi$ of a system of $A$ particles is generally a function of $A$ coordinates of individual particles $\boldsymbol{r}_{i}$. The center of mass motion can be separated and eliminated; as a result we can treat the wave function $\Psi$ as a function of $A-1$ Jacobi coordinates $\boldsymbol{\xi}_{i}$. Within the democratic decay approximation, it is natural to employ the hyperspherical harmonics formalism (see, e. g., $[15,28]$ ). The $3 A-3$ independent variables $\boldsymbol{\xi}_{i}$ are rearranged in this

formalism: the so-called hyperradius $\rho=\sqrt{\sum_{i=1}^{A-1} \xi_{i}^{2}}$ is introduced and all the rest $3 A-4$ variables are the angles on the $(3 A-3)$-dimensional sphere. The wave function is searched for in the form

$$
\Psi=\sum_{\Gamma} d_{\Gamma} \Psi_{\Gamma}
$$

where

$$
\Psi_{\Gamma} \equiv \Psi_{K \gamma}=\Phi_{K \gamma}(\rho) \mathscr{Y}_{K \gamma}(\Omega),
$$

$\Omega$ is the set of angles on the $(3 A-3)$-dimensional sphere, $K$ is the so-called hypermomentum [physically $K$ is the angular momentum in the $(3 A-3)$ dimensional space; $K \geq L$ where $L$ is the orbital angular momentum] and $\gamma$ stands for all the rest quantum numbers labelling the hyperspherical function $\mathscr{Y}_{K \gamma}(\Omega)$, the multi-index $\Gamma=\{K, \gamma\}$. Various analytic expressions may be found for the hyperspherical functions $\mathscr{Y}_{K \gamma}(\Omega)$ in textbooks (see, e. g., [15]).

In the two-body case, $\rho=r=\left|\boldsymbol{r}_{1}-\boldsymbol{r}_{2}\right|$ is the distance between the particles and $\mathscr{Y}_{K \gamma}(\Omega)$ becomes the usual spherical function $Y_{L M}(\Omega)$ with $K$ and $\gamma$ playing the role of the angular momentum $L$ and its projection $M$, respectively. Therefore equation (2) is a generalization on the $A$-body $(A \geq 3)$ case of the conventional wave function used in the study of spherically symmetric twobody systems. However, contrary to the case of two-body systems with central interaction which impose the spherical symmetry, the hypermomentum $K$ is not an integral of motion: the two-body interactions in the $A$-body system couples the states with different values of the hypermomentum $K$ and we have a set of coupled equations for the hyperradial functions $\Phi_{K \gamma}(\rho)$. This set of equations has the same structure as the one describing two-body systems with non-central forces; from the point of view of scattering theory, this set of coupled equations is formally equivalent to the one describing multichannel scattering in the system with the hyperspherical channels $\Gamma$.

We introduce the hyperspherical oscillator basis

$$
|\kappa \Gamma\rangle \equiv|\kappa K \gamma\rangle=\mathscr{R}_{\kappa K}(\rho) \mathscr{Y}_{K \gamma}(\Omega),
$$


where the hyperradial oscillator function

$$
\begin{aligned}
& \mathscr{R}_{\kappa K}(\rho) \equiv \mathscr{R}_{\kappa}^{\mathscr{L}}(\rho)=\rho^{-(3 A-4) / 2} \mathfrak{r}_{\kappa K}(\rho), \\
& \mathfrak{r}_{\kappa K}(\rho) \equiv \mathfrak{r}_{\kappa}^{\mathscr{L}}(\rho)=(-1)^{\kappa} \sqrt{\frac{2 \kappa !}{\Gamma(\kappa+\mathscr{L}+3 / 2)}} \rho^{\mathscr{L}+1} e^{-\rho^{2} / 2} L_{\kappa}^{\mathscr{L}+\frac{1}{2}}\left(\rho^{2}\right),
\end{aligned}
$$

$L_{n}^{\alpha}(x)$ is the associated Laguerre polynomial and

$$
\mathscr{L}=K+\frac{3 A-6}{2} .
$$

In the two-body case $(A=2), \mathscr{L}$ is equivalent to the orbital angular momentum $L, \mathscr{L}=L$, and Eqs. (3)-(6) define the conventional oscillator basis. In the many-body case $(A \geq 3)$, Eqs. (3)-(6) define the $A$-body oscillator functions, i. e. the eigenfunctions of the $A$-body Schrödinger equation with the potential energy

$$
U=\frac{\omega^{2}}{2} \sum_{i=1}^{A} m_{i}\left(\boldsymbol{r}_{i}-\boldsymbol{R}\right)^{2}=\frac{\hbar \omega}{2} \rho^{2},
$$

where $m_{i}$ is the mass of the $i$ th particle, $\boldsymbol{R}$ is the center-of-mass coordinate and $\omega$ is the parameter of the oscillator basis. The corresponding eigenenergy is

$$
E_{\kappa K}=\left(N+\frac{3 A-3}{2}\right) \hbar \omega
$$

where the number of oscillator quanta

$$
N=2 \kappa+K=2 \kappa+\mathscr{L}-\frac{3 A-6}{2} .
$$

The hyperspherical oscillator basis (3) is orthonormalized:

$$
\left\langle\kappa K \gamma \mid \kappa^{\prime} K^{\prime} \gamma^{\prime}\right\rangle=\delta_{\kappa \kappa^{\prime}} \delta_{\Gamma \Gamma^{\prime}}
$$

or

$$
\int_{0}^{\infty} \mathfrak{r}_{\kappa K}^{*}(\rho) \mathfrak{r}_{\kappa^{\prime} K}(\rho) \mathrm{d} \rho=\delta_{\kappa \kappa^{\prime}}
$$

The wave function $\Psi$ is expanded in the hyperspherical oscillator function series,

$$
\Psi=\sum_{\kappa, K, \gamma}\langle\kappa K \gamma \mid \Psi\rangle|\kappa K \gamma\rangle
$$

and the Schrödinger equation takes the form

$$
\sum_{\kappa^{\prime}, K^{\prime}, \gamma^{\prime}}\left\langle\kappa K \gamma|H-E| \kappa^{\prime} K^{\prime} \gamma^{\prime}\right\rangle\left\langle\kappa^{\prime} K^{\prime} \gamma^{\prime} \mid \Psi\right\rangle=0
$$


where $E$ is the energy, the Hamiltonian $H=T+V$, the potential energy $V$ is usually a superposition of two-body interactions $V_{i j}, V=\sum_{i<j} V_{i j}$, and $T$ is the $A$-body kinetic energy operator. Generally, the Hamiltonian matrix $\left\langle\kappa K \gamma|H| \kappa^{\prime} K^{\prime} \gamma^{\prime}\right\rangle$ is infinite. However, within the $J$-matrix formalism we truncate the potential energy matrix; it is most natural to define the truncation boundary through the number of oscillator quanta, i. e. we suppose that

$$
\left\langle\kappa K \gamma|V| \kappa^{\prime} K^{\prime} \gamma^{\prime}\right\rangle=0 \quad \text { if } \quad 2 \kappa+K>\tilde{N} \quad \text { or } \quad 2 \kappa^{\prime}+K^{\prime}>\tilde{N}
$$

The kinetic energy matrix $\left\langle\kappa K \gamma|T| \kappa^{\prime} K^{\prime} \gamma^{\prime}\right\rangle$ is tridiagonal,

$$
\begin{aligned}
\left\langle\kappa K \gamma|T| \kappa^{\prime} K^{\prime} \gamma^{\prime}\right\rangle= & \frac{\hbar \omega}{2} \delta_{K K^{\prime}} \delta_{\gamma \gamma^{\prime}}\left[-\sqrt{(n+1)\left(n+\mathscr{L}+\frac{3}{2}\right)} \delta_{\kappa+1, \kappa^{\prime}}\right. \\
& \left.+\left(2 n+\mathscr{L}+\frac{3}{2}\right) \delta_{\kappa \kappa^{\prime}}-\sqrt{n\left(n+\mathscr{L}+\frac{1}{2}\right)} \delta_{\kappa-1, \kappa^{\prime}}\right] .
\end{aligned}
$$

The $A$-body hyperspherical $J$-matrix formalism is very close to the conventional $J$-matrix formalism with oscillator basis $[2,29]$ used in multichannel two-body problems. The main difference is that $\mathscr{L}$ entering Eq. (15) is halfinteger if the number of particles $A$ is odd [see (6) and note that $K$ is always integer], in particular, $\mathscr{L}$ is half-integer in the three-body case discussed here. The general $J$-matrix oscillator-basis solutions of the free Schrödinger equation

$$
\sum_{\kappa^{\prime}, K^{\prime}, \gamma^{\prime}}\left\langle\kappa K \gamma|T-E| \kappa^{\prime} K^{\prime} \gamma^{\prime}\right\rangle\left\langle\kappa^{\prime} K^{\prime} \gamma^{\prime} \mid \Psi\right\rangle=0
$$

that may be used in the case of arbitrary $\mathscr{L}$, were suggested in Ref. [20]. The regular solution is

$$
S_{\kappa K}(q)=\sqrt{\frac{2 \kappa !}{\Gamma(n+\mathscr{L}+3 / 2)}} q^{\mathscr{L}+1} e^{-q^{2} / 2} L_{\kappa}^{\mathscr{L}+\frac{1}{2}}\left(q^{2}\right)
$$

the irregular solutions are

$$
\begin{aligned}
& C_{\kappa K}(q)=-\frac{2 q}{\pi S_{0 K}(q)} \mathbf{V} . \mathbf{P} \cdot \int_{0}^{\infty} \frac{S_{0 K}\left(q^{\prime}\right) S_{\kappa K}\left(q^{\prime}\right)}{q^{2}-q^{\prime 2}} d q^{\prime}, \\
& C_{\kappa K}^{(+)}(q)=-\frac{2 q}{\pi S_{0 K}(q)} \int_{0}^{\infty} \frac{S_{0 K}\left(q^{\prime}\right) S_{\kappa K}\left(q^{\prime}\right)}{q^{2}-q^{2}+i 0} d q^{\prime}, \\
& C_{\kappa K}^{(-)}(q)=-\frac{2 q}{\pi S_{0 K}(q)} \int_{0}^{\infty} \frac{S_{0 K}\left(q^{\prime}\right) S_{\kappa K}\left(q^{\prime}\right)}{q^{2}-q^{2}-i 0} d q^{\prime},
\end{aligned}
$$


where $q=\sqrt{\frac{2 E}{\hbar \omega}}, \mathbf{V}$. P. in Eq. (18) indicates the principal value integral, $+i 0$ and $-i 0$ in Eqs. (19)-(20) show how the poles of the integrand should be treated. The solutions (17)-(20) are not independent, the following relation between them is valid:

$$
C_{\kappa K}^{( \pm)}(q)=C_{\kappa K}(q) \pm i S_{\kappa K}(q)
$$

However, any pair of the solutions (17)-(20) can be used to construct an arbitrary solution.

The regular solution (17) is just the hyperradial momentum-space oscillator function. The irregular solutions (18)-(20) are more complicated. They were analyzed in detail in Ref. [20]. In the general case, they can be expressed through Tricomi function. However in the case of even $A$, the irregular solutions can be simplified and expressed through the confluent hypergeometric function. The physical meaning of the solutions (17)-(20) is clear from the following expressions [20]:

$$
\begin{aligned}
& \sum_{\kappa=0}^{\infty} S_{\kappa K}(q) \mathfrak{r}_{\kappa K}(\rho)=\sqrt{q \rho} J_{\mathscr{L}+\frac{1}{2}}(q \rho) \underset{\rho \rightarrow \infty}{\longrightarrow} \sqrt{\frac{2}{\pi}} \sin \left(q \rho-\frac{\pi \mathscr{L}}{2}\right) \\
& \sum_{\kappa=0}^{\infty} C_{\kappa K}(q) \mathfrak{r}_{\kappa K}(\rho) \underset{\rho \rightarrow \infty}{\longrightarrow}-\sqrt{q \rho} N_{\mathscr{L}+\frac{1}{2}}(q \rho) \underset{\rho \rightarrow \infty}{\longrightarrow} \sqrt{\frac{2}{\pi}} \cos \left(q \rho-\frac{\pi \mathscr{L}}{2}\right) \\
& \sum_{\kappa=0}^{\infty} C_{\kappa K}^{(+)}(q) \mathfrak{r}_{\kappa K}(\rho) \underset{\rho \rightarrow \infty}{\longrightarrow} i \sqrt{q \rho} H_{\mathscr{L}+\frac{1}{2}}^{(1)}(q \rho) \underset{\rho \rightarrow \infty}{\longrightarrow} \sqrt{\frac{2}{\pi}} e^{i\left(q \rho-\frac{\pi \mathscr{L}}{2}\right)}, \\
& \sum_{\kappa=0}^{\infty} C_{\kappa K}^{(-)}(q) \mathfrak{r}_{\kappa K}(\rho) \underset{\rho \rightarrow \infty}{\longrightarrow}-i \sqrt{q \rho} H_{\mathscr{L}+\frac{1}{2}}^{(2)}(q \rho) \underset{\rho \rightarrow \infty}{\longrightarrow} \sqrt{\frac{2}{\pi}} e^{-i\left(q \rho-\frac{\pi \mathscr{L}}{2}\right)} .
\end{aligned}
$$

Here $J_{\alpha}(x), N_{\alpha}(x)$ and $H_{\alpha}^{(1,2)}(x)$ are Bessel, Neumann and Hankel functions, respectively.

In the case of continuum spectrum $(E>0)$, the oscillator representation wave function $\left\langle\kappa K \gamma \mid \Psi^{\left(\Gamma^{\prime}\right)}\right\rangle \equiv\left\langle\kappa \Gamma \mid \Psi^{\left(\Gamma^{\prime}\right)}\right\rangle$ in the channel $\Gamma$ is of the form

$$
\left\langle\kappa \Gamma \mid \Psi^{\left(\Gamma^{\prime}\right)}\right\rangle=\frac{1}{2}\left(\delta_{\Gamma \Gamma^{\prime}} C_{\kappa K}^{(-)}(q)-C_{\kappa K}^{(+)}(q)[\mathbf{S}]_{\Gamma \Gamma^{\prime}}\right)
$$

in the 'external region' $\kappa \geq \kappa_{\Gamma}$, where $\kappa_{\Gamma}=(\tilde{N}-K) / 2$ is the potential energy truncation boundary in the channel $\Gamma$. It is supposed that the ingoing spherical wave is present in the channel $\Gamma^{\prime}$ only while the outgoing spherical waves are present in all channels; $[\mathbf{S}]_{\Gamma \Gamma^{\prime}}$ entering Eq. (26) is the matrix element of the hyperspherical $S$-matrix. The $S$-matrix $[\mathbf{S}$ ] can be calculated by the following formula [20]:

$$
[\mathbf{S}]=\left[\mathbf{A}^{s}\right]^{-1}\left[\mathbf{B}^{s}\right]
$$


where the matrix elements of the matrices $\left[\mathbf{A}^{s}\right]$ and $\left[\mathbf{B}^{s}\right]$ are

$$
\begin{aligned}
& {\left[\mathbf{A}^{s}\right]_{\Gamma^{\prime} \Gamma}=\left\langle\kappa_{\Gamma^{\prime}} \Gamma^{\prime}|\mathscr{P}| \kappa_{\Gamma}+1, \Gamma\right\rangle C_{\kappa_{\Gamma}+1, K}^{(+)}(q)-\delta_{\Gamma \Gamma^{\prime}} C_{\kappa_{\Gamma} K}^{(+)}(q),} \\
& {\left[\mathbf{B}^{s}\right]_{\Gamma^{\prime} \Gamma}=\left\langle\kappa_{\Gamma^{\prime}} \Gamma^{\prime}|\mathscr{P}| \kappa_{\Gamma}+1, \Gamma\right\rangle C_{\kappa_{\Gamma}+1, K}^{(-)}(q)-\delta_{\Gamma \Gamma^{\prime}} C_{\kappa_{\Gamma} K}^{(-)}(q)}
\end{aligned}
$$

the matrix elements of the matrix $[\mathscr{P}]$ proportional to the discrete analog of the $P$-matrix (see Ref. [29] for details) are

$$
\left\langle\kappa \Gamma|\mathscr{P}| \kappa_{\Gamma^{\prime}}+1, \Gamma^{\prime}\right\rangle=\left\langle\kappa \Gamma|\mathfrak{P}| \kappa_{\Gamma^{\prime}} \Gamma^{\prime}\right\rangle\left\langle\kappa_{\Gamma^{\prime}} \Gamma^{\prime}|T| \kappa_{\Gamma^{\prime}}+1, \Gamma^{\prime}\right\rangle,
$$

the kinetic energy matrix elements $\left\langle\kappa_{\Gamma^{\prime}} \Gamma^{\prime}|T| \kappa_{\Gamma^{\prime}}+1, \Gamma^{\prime}\right\rangle$ are given by Eq. (15), and the matrix $[\mathfrak{P}] \equiv[H-E]^{-1}$ defined in the truncated model space spanned by oscillator functions (3) with $\kappa \leq \kappa_{\Gamma}=(\tilde{N}-K) / 2$ in each channel $\Gamma$, has the matrix elements

$$
\left\langle\kappa \Gamma|\mathfrak{P}| \kappa^{\prime} \Gamma^{\prime}\right\rangle=\sum_{\lambda} \frac{\langle\kappa \Gamma \mid \lambda\rangle\left\langle\lambda \mid \kappa^{\prime} \Gamma^{\prime}\right\rangle}{E-E_{\lambda}} .
$$

In Eq. (31), $E_{\lambda}$ are eigenenergies and $\langle\kappa \Gamma \mid \lambda\rangle$ are the respective eigenvectors of the truncated Hamiltonian matrix, i. e. $E_{\lambda}$ and $\langle\kappa \Gamma \mid \lambda\rangle$ can be found by solving Eq. (13) supposing that $\kappa \leq \kappa_{\Gamma}$ and $\kappa^{\prime} \leq \kappa_{\Gamma^{\prime}}$. The results obtained by diagonalization of the truncated Hamiltonian matrix we shall refer to as variational results.

After performing variational calculation, we obtain the $S$-matrix with the help of Eqs. (27)-(31) and the oscillator representation wave function $\left\langle\kappa \Gamma \mid \Psi^{\left(\Gamma^{\prime}\right)}\right\rangle$ in the external region $\kappa \geq \kappa_{\Gamma}$ by the formula (26). The oscillator representation wave function $\left\langle\kappa \Gamma \mid \Psi^{\left(\Gamma^{\prime}\right)}\right\rangle$ in the 'internal region' $\kappa \leq \kappa_{\Gamma}$ can be now calculated as

$$
\left\langle\kappa \Gamma \mid \Psi^{\left(\Gamma^{\prime}\right)}\right\rangle=\sum_{\Gamma^{\prime \prime}}\left\langle\kappa \Gamma|\mathscr{P}| \kappa_{\Gamma^{\prime \prime}}+1, \Gamma^{\prime \prime}\right\rangle\left\langle\kappa_{\Gamma^{\prime \prime}}+1, \Gamma^{\prime \prime} \mid \Psi^{\left(\Gamma^{\prime}\right)}\right\rangle, \quad \kappa \leq \kappa_{\Gamma} .
$$

Equation (27) can be used in the complex momentum plane; the bound state energies are associated with the $S$-matrix poles that can be found by the numerical solution of the obvious equation

$$
\operatorname{det}\left[\mathbf{A}^{s}\right]=0
$$

The matrix $\left[\mathbf{A}^{s}\right]$ in Eq. (33) is the extension on the complex momentum plane of (28); for the the bound states with $E=\frac{1}{2} q^{2} \hbar \omega<0$ its matrix elements are

$$
\left[\mathbf{A}^{s}\right]_{\Gamma^{\prime} \Gamma}=\left\langle\kappa_{\Gamma^{\prime}} \Gamma^{\prime}|\mathscr{P}| \kappa_{\Gamma}+1, \Gamma\right\rangle C_{\kappa_{\Gamma}+1, K}^{(b)}(q)-\delta_{\Gamma \Gamma^{\prime}} C_{\kappa_{\Gamma} K}^{(b)}(q),
$$

where

$$
C_{\kappa K}^{(b)}(q)=C_{\kappa K}^{(+)}(i|q|)
$$


The relation (26) for the oscillator representation wave function in the external region $\kappa \geq \kappa_{\Gamma}$ should be replaced for bound states by

$$
\langle\kappa \Gamma \mid \Psi\rangle=-[\mathbf{S}]_{\Gamma \Gamma} C_{\kappa K}^{(b)}(q)
$$

the multipliers $[\mathbf{S}]_{\Gamma г}$ are obtained by the numerical solution of the equation

$$
[\mathbf{S}]_{\Gamma \Gamma} C_{\kappa_{\Gamma} K}^{(b)}(q)=\sum_{\Gamma^{\prime}}\left\langle\kappa_{\Gamma} \Gamma|\mathscr{P}| \kappa_{\Gamma^{\prime}}+1, \Gamma^{\prime}\right\rangle[\mathbf{S}]_{\Gamma^{\prime} \Gamma^{\prime}} C_{\kappa_{\Gamma^{\prime}}+1, K}^{(b)}(q)
$$

[we note that Eq. (33) is the condition of solvability of Eq. (37) for the bound states]. Equation (32) can be used for the calculation of the bound state oscillator representation wave function $\left\langle\kappa \Gamma \mid \Psi^{\left(\Gamma^{\prime}\right)}\right\rangle$ in the internal region $\kappa \leq \kappa_{\Gamma}$. Since the set of $[\mathbf{S}]_{\Gamma \Gamma}$ can be obtained from Eq. (37) up to a common multiplier only, the bound state wave function should be normalized numerically.

The bound state energies and wave functions obtained by numerical calculation of the $S$-matrix poles, will be refered to as $J$-matrix results.

In practical applications, it is useless to allow for all possible channels $\Gamma=$ $\{K, \gamma\}$ in the external region $N \equiv 2 \kappa+K>\tilde{N}$. We start the calculations allowing for the channels $\Gamma$ with few minimal possible values of $K$ in the external region only, i. e. we allow for the channels with all possible values of $K \leq K_{\mathrm{tr}}$ and all possible $\gamma$ values for a given $K$ in the external region. Therefore the summation over $\Gamma^{\prime \prime}$ in $(32)$ is restricted to these allowed channels. However all possible channels with $K \leq \tilde{N}$ are allowed for in the internal region $N \equiv 2 \kappa+K \leq \tilde{N}$. As a next step, we increase the value of $K_{\text {tr }}$ and allow for more channels $\Gamma$ in the external region. The convergence of all results (binding energies and other bound state observables, transition probabilities, etc.) with $K_{\text {tr }}$ is carefully examined. The convergence is usually achieved at small enough values of $K_{\text {tr }}$ (much less than $\tilde{N}$ ). Such converged with respect to $K_{\text {tr }}$ results are discussed below.

\section{Application to ${ }^{11} \mathrm{Li}$ nucleus}

The ${ }^{11} \mathrm{Li}$ nucleus is studied in the three-body cluster model ${ }^{9} \mathrm{Li}+n+n$. The three-body cluster model for ${ }^{11} \mathrm{Li}$ was first suggested in Ref. [30]. The ${ }^{11} \mathrm{Li}$ three-body binding energy or equivalently the ${ }^{11} \mathrm{Li}$ two-neutron separation energy is $0.295 \pm 0.035 \mathrm{MeV}$ [31] that is much less than the excitation energy

of the lowest excited state in the cluster ${ }^{9} \mathrm{Li}$. As it was already noted, ${ }^{11} \mathrm{Li}$ is a Borromean nuclei, i. e. none of the two-body subsystems ${ }^{9} \mathrm{Li}+n$ and $n+n$ has a bound state.

The two-body potentials are needed for the investigation of the three-body 
system ${ }^{11} \mathrm{Li}={ }^{9} \mathrm{Li}+n+n$. Unfortunately the information about the $n-{ }^{9} \mathrm{Li}$ interaction is scarce. As a result, in Ref. [30] the $n-{ }^{9} \mathrm{Li}$ potential was phenomenologically parametrized and fitted to the ${ }^{11} \mathrm{Li}$ binding energy. Therefore it is reasonable to use a simplified three-body model with simplified interactions to avoid computational difficulties; a microscopic extension of the model seems to be useless due to uncertainties of the $n-{ }^{9} \mathrm{Li}$ interaction.

We employ the $n-n$ and $n-{ }^{9} \mathrm{Li}$ potentials of Ref. [30]. The Gaussian $n-n$ potential

$$
U(r)=-V_{0} e^{-(r / R)^{2}}
$$

was fitted to the low-energy singlet $(S=0) s$ wave phase shifts, its depth $V_{0}=31 \mathrm{MeV}$ and its width $R=1.8 \mathrm{fm}$. The $n-{ }^{9} \mathrm{Li}$ potential is of a twoGaussian form,

$$
U(r)=-V_{1} e^{-\left(r / R_{1}\right)^{2}}-V_{2} e^{-\left(r / R_{2}\right)^{2}},
$$

with $V_{1}=7 \mathrm{MeV}, R_{1}=2.4 \mathrm{fm}, V_{2}=1 \mathrm{MeV}$, and $R_{2}=3.0 \mathrm{fm}$. It is supposed that two valent neutrons in ${ }^{11} \mathrm{Li}$ are in a singlet spin state. The total angular momentum $J$ in ${ }^{11} \mathrm{Li}$ results from the coupling of the spin $\frac{3}{2}$ of the ${ }^{9} \mathrm{Li}$ cluster with the relative motion orbital angular momentum $L$; however we do not make use of the spin-flip operators in our investigation, therefore we can disregard the spin variables and exclude them from the wave function. The ${ }^{11} \mathrm{Li}$ ground state orbital angular momentum $L$ is supposed to be zero. We can define the channels $\Gamma$ as $\Gamma=\left\{K, l_{x}, l_{y}, L\right\}$ where $l_{x}$ and $l_{y}$ are orbital angular momenta corresponding to the Jacobi coordinates

$$
\begin{aligned}
\boldsymbol{x} & =\sqrt{\frac{\omega}{\hbar} \frac{m_{1} m_{2}}{m_{1}+m_{2}}}\left(\boldsymbol{r}_{1}-\boldsymbol{r}_{2}\right), \\
\boldsymbol{y} & =\sqrt{\frac{\omega}{\hbar} \frac{m_{3}\left(m_{1}+m_{2}\right)}{m_{1}+m_{2}+m_{3}}}\left(\frac{m_{1} \boldsymbol{r}_{1}+m_{2} \boldsymbol{r}_{2}}{m_{1}+m_{2}}-\boldsymbol{r}_{3}\right)
\end{aligned}
$$

the angular momenta $l_{x}$ and $l_{y}$ are coupled to the orbital angular momentum $L$. The wave function is given by the general formula (12). The oscillator basis (3) in our case takes the form

$$
|\kappa \Gamma\rangle \equiv\left|\kappa K l_{x} l_{y}: L M\right\rangle \equiv\left\langle\boldsymbol{\rho} \mid \kappa K l_{x} l_{y}: L M\right\rangle=\mathscr{R}_{\kappa, K}(\rho) \mathscr{Y}_{K \gamma}(\Omega)
$$

where the hyperspherical functions

$$
\begin{aligned}
\mathscr{Y}_{K \gamma}(\Omega) \equiv \mathscr{Y}_{K l_{x} l_{y} L M}(\Omega) & \\
=N_{K}^{l_{x} l_{y}} & \cos ^{l_{x}} \alpha \sin ^{l_{y}} \alpha P_{n}^{l_{y}+1 / 2, l_{x}+1 / 2}(\cos 2 \alpha) \\
& \times \sum_{m_{x}+m_{y}=M}\left(l_{x} m_{x} l_{y} m_{y} \mid L M\right) Y_{l_{x}, m_{x}}(\hat{\boldsymbol{x}}) Y_{l_{y}, m_{y}}(\hat{\boldsymbol{y}}),
\end{aligned}
$$

$\alpha=\tan ^{-1}(y / x), Y_{l m}$ is the spherical function, $\left(l_{x} m_{x} l_{y} m_{y} \mid L M\right)$ is the ClebschGordan coefficient, $P_{n}^{\alpha, \beta}(x)$ is Jacobi polynomial [32], and the normalization 
factor

$$
N_{K}^{l_{x} l_{y}}=\sqrt{\frac{2 n !(K+2)\left(n+l_{x}+l_{y}\right) !}{\Gamma\left(n+l_{x}+3 / 2\right) \Gamma\left(n+l_{y}+3 / 2\right)}} .
$$

The basis (42) is useful for the calculation of three-body decays. However it is not convenient in the calculation of the matrix elements of two-body potentials $V_{i j}$. These matrix elements can be easily calculated in the basis

$$
\begin{aligned}
\left|n_{x} l_{x} n_{y} l_{y}: L M\right\rangle \equiv & \left\langle\boldsymbol{x} \boldsymbol{y} \mid n_{x} l_{x} n_{y} l_{y}: L M\right\rangle \\
& =\sum_{m_{x}+m_{y}=M}\left(l_{x} m_{x} l_{y} m_{y} \mid L M\right)\left\langle\boldsymbol{x} \mid n_{x} l_{x} m_{x}\right\rangle\left\langle\boldsymbol{y} \mid n_{y} l_{y} m_{y}\right\rangle
\end{aligned}
$$

where $\left\langle\boldsymbol{x} \mid n_{x} l_{x} m_{x}\right\rangle$ and $\left\langle\boldsymbol{y} \mid n_{y} l_{y} m_{y}\right\rangle$ are the conventional three-dimensional oscillator functions depending on the Jacobi coordinates $\boldsymbol{x}$ and $\boldsymbol{y}$, respectively. The unitary transformations relating the basises (42) and (44),

$$
\begin{gathered}
\left|\kappa K l_{x} l_{y}: L M\right\rangle=\sum_{n_{x}, n_{y}}\left\langle n_{x} l_{x} n_{y} l_{y} \mid \kappa K\right\rangle\left|n_{x} l_{x} n_{y} l_{y}: L M\right\rangle, \\
\left|n_{x} l_{x} n_{y} l_{y}: L M\right\rangle=\sum_{\kappa, K}\left\langle n_{x} l_{x} n_{y} l_{y} \mid \kappa K\right\rangle\left|\kappa K l_{x} l_{y}: L M\right\rangle
\end{gathered}
$$

are discussed in detail in Refs. [15,28]. The transformation coefficients $\left\langle n_{x} l_{x} n_{y} l_{y} \mid \kappa K\right\rangle$ are non-zero if only the the basis functions (42) and (44) are characterized by the same number of oscillator quanta $N$, i. e. when $2 n_{x}+l_{x}+2 n_{y}+l_{y}=2 \kappa+K$.

We suppose that the 1st and the 2nd particles are neutrons and the 3rd particle is the ${ }^{9} \mathrm{Li}$ cluster (or the ${ }^{4} \mathrm{He}$ cluster in the next section). Therefore the Jacobi coordinate $\boldsymbol{x}$ is proportional to the distance between the valent neutrons and the Jacobi coordinate $\boldsymbol{y}$ is proportional to the distance between the cluster and the center of mass of two valent neutrons. In this case the basis (44) can be used directly for the calculation of the $n-n$ interaction matrix elements. For the calculation of the matrix elements of the $n$-cluster interaction, we renumerate the particles assigning number 1 to the cluster and numbers 2 and 3 to the valent neutrons, and use Eqs. (40)-(41) to define another set of Jacobi coordinates $\boldsymbol{x}^{\prime}$ and $\boldsymbol{y}^{\prime}$. The $n$-cluster interaction matrix elements can be directly calculated now in the basis (44). The unitary transformation of the wave functions associated with the switching from one set of Jacobi coordinates $(\boldsymbol{x}$ and $\boldsymbol{y}$ ) to another $\left(\boldsymbol{x}^{\prime}\right.$ and $\left.\boldsymbol{y}^{\prime}\right)$, is discussed in detail in Refs. [15,28].

The ${ }^{11} \mathrm{Li}$ ground state energy dependence on the oscillator basis parameter $\hbar \omega$ is shown on Fig. 1 for different values for the truncation boundary $\tilde{N}$. The dependence is typical for variational calculations and has a minimum at $\hbar \omega \approx 6.5 \mathrm{MeV}$ (the best convergence $\hbar \omega$ value). The $\hbar \omega$ ground state energy dependence obtained in variational and $J$-matrix calculations are of 

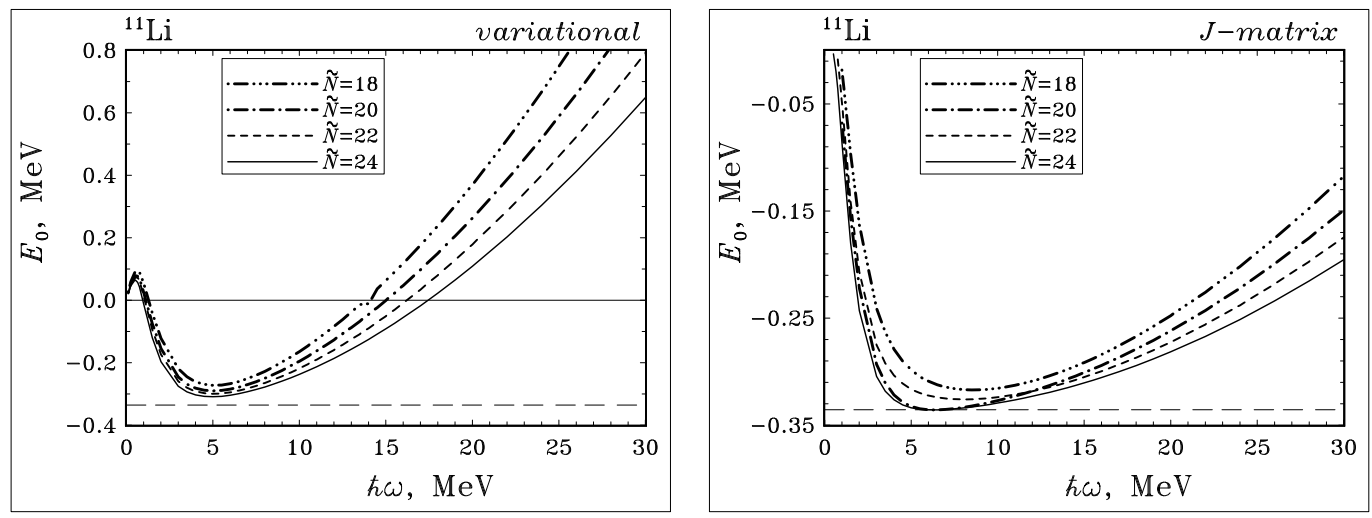

Fig. 1. The ${ }^{11} \mathrm{Li}$ ground state energy vs the oscillator basis parameter $\hbar \omega$ in the variational (left panel) and $J$-matrix (right panel) calculations for different values of the truncation boundary $\tilde{N}$. The horizontal dashed line depicts the convergence limit for the ground state energy.

the same type. However this dependence is much less pronounced in the $J$ matrix calculations (note a different scale on the left and right panels of Fig. 1). Therefore the $J$-matrix calculation is much less sensitive to the choice of the $\hbar \omega$ value. The $J$-matrix ground state energy results are better than the variational ones for any value of $\hbar \omega$ and any value of $\tilde{N}$.

This is clearly seen on Fig. 2 where we present the convergence with $\tilde{N}$ of the ${ }^{11} \mathrm{Li}$ ground state energy and rms matter radius

$$
\left\langle r^{2}\right\rangle^{1 / 2}=\sqrt{\frac{A-2}{A}\left\langle r_{c}^{2}\right\rangle+\left\langle\rho^{2}\right\rangle},
$$

where the ${ }^{11} \mathrm{Li}$ mass number $A=11$ and $\left\langle r_{c}^{2}\right\rangle^{1 / 2}$ is the rms radius of the ${ }^{9} \mathrm{Li}$ cluster. The variational ground state energy decreases monotonically due to the variational principal; the variational rms radius consequently monotonically increases. We note here that the variational principle is not applicable to the $J$-matrix calculations where the infinite oscillator basis is allowed for and the results obtained with different $\tilde{N}$ values differ by the rank of the potential energy matrix. The $J$-matrix results demonstrate a much more interesting dependence on $\tilde{N}$ than the variational ones. For the $\hbar \omega$ values close to the best convergence value of $\hbar \omega=6.5 \mathrm{MeV}$, there is a staggering of the $J$-matrix binding energy and rms radius with $\tilde{N}$ (Fig. 2, left panel). The amplitude of the staggering decreases with $\tilde{N}$ and the results converge rapidly. It is interesting that the binding energy and the rms radius converge to the values lying between the results obtained with two subsequent values of $\tilde{N}$. Hence we obtain not only the lower bound binding energy and rms radius estimates as in variational calculations but both lower bound and upper bound estimates for these observables. These estimates are presented in Table 1 together with experimental data and the results of calculations of other authors. 

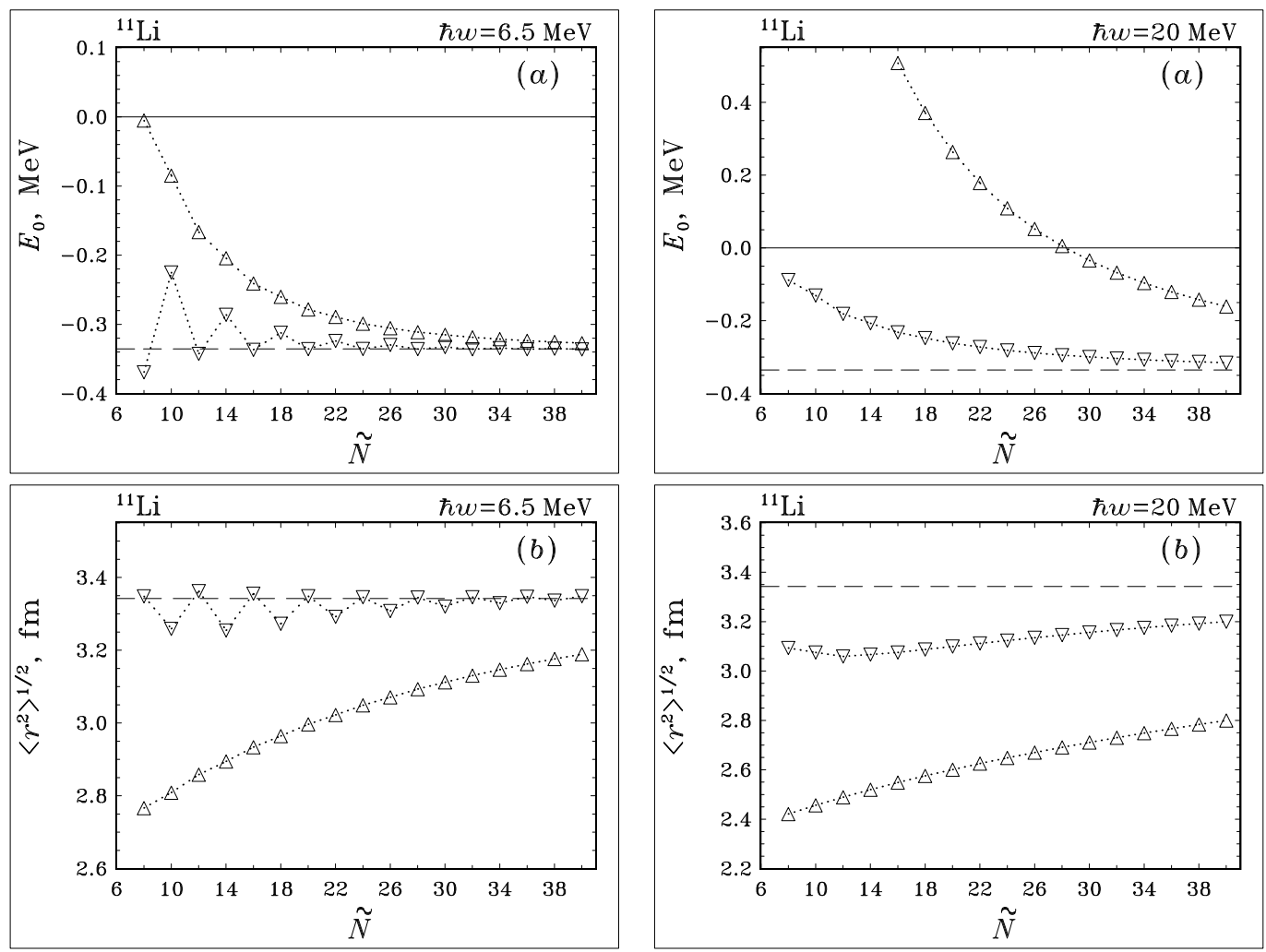

Fig. 2. Convergence with $\tilde{N}$ of the ${ }^{11} \mathrm{Li}$ three-body ground state energy (a) and rms matter radius (b) for $\hbar \omega=6.5 \mathrm{MeV}$ (left panel) and $\hbar \omega=20 \mathrm{MeV}$ (right panel). Variational and $J$-matrix results are shown by triangles up and triangles down, respectively. The convergence limits are shown by the dashed line.

Table 1

The ${ }^{11} \mathrm{Li}$ two-neutron separation energy $E(2 n)$ and rms matter radius $\left\langle r^{2}\right\rangle^{1 / 2}$ obtained in the calculations with $\hbar \omega=6.5 \mathrm{MeV}$ together with the results of theoretical studies of Ref. [33] and experimental data.

\begin{tabular}{|c|cc|cc|}
\hline \multirow{2}{*}{ Approximation } & \multicolumn{2}{|c|}{$E(2 n), \mathrm{MeV}$} & \multicolumn{2}{|c|}{$\left\langle r^{2}\right\rangle^{1 / 2}, \mathrm{fm}$} \\
& $\tilde{N}=38$ & $\tilde{N}=40$ & $\tilde{N}=38$ & $\tilde{N}=40$ \\
\hline variational & 0.326 & 0.327 & 3.176 & 3.189 \\
$J$-matrix & 0.335 & 0.336 & 3.336 & 3.348 \\
Ref. [33] & \multicolumn{2}{|c|}{0.3} & 3.32 \\
\hline \multirow{2}{*}{ experimental } & $0.247 \pm 0.080$, Ref. [34] & $3.10 \pm 0.17$, Ref. [35] \\
data & $0.295 \pm 0.035$, Ref. [31] & $3.53 \pm 0.10$, Ref. [36] \\
& & $3.55 \pm 0.10$, Ref. [37] \\
\hline
\end{tabular}




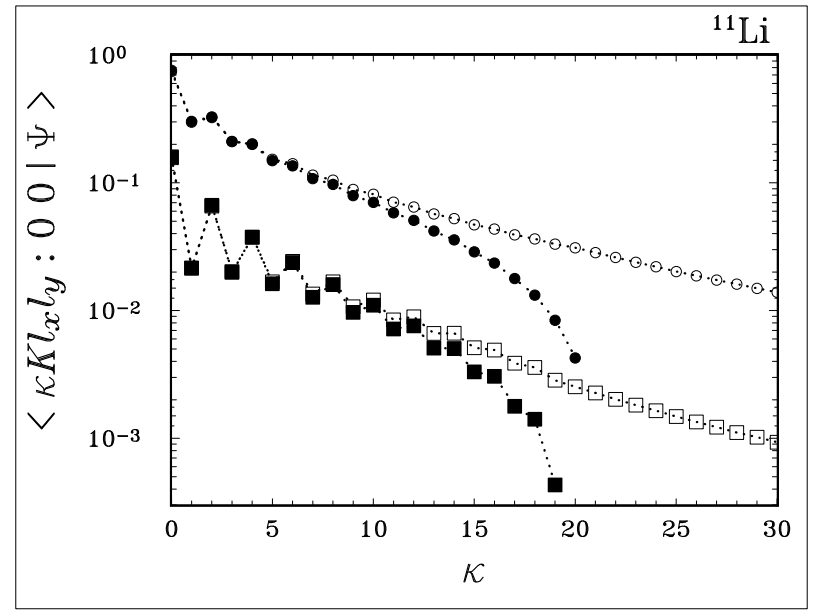

Fig. 3. The dominant $K=0$ and $K=2, l_{x}=l_{y}=0$ components of the ${ }^{11} \mathrm{Li}$ ground state wave function in the oscillator representation, $\left\langle\kappa, K=0, l_{x}=0, l_{y}=0: 00 \mid \Psi\right\rangle$ (circles) and $\left\langle\kappa, K=2, l_{x}=0, l_{y}=0: 00 \mid \Psi\right\rangle$ (squares), obtained in the calculations with $\tilde{N}=40$ and $\hbar \omega=6.5 \mathrm{MeV}$. The bold and empty symbols are the variational and the $J$-matrix results, respectively.

If $\hbar \omega$ differs much from the best convergence value, we have the conventional variational-type monotonic convergence of the $J$-matrix ground state energy and rms radius (Fig. 2, right panel). The results converge slower with $\tilde{N}$ in this case, however much faster than in the variational calculation.

The structure of the ground state wave function is the following. The total weight of the $l_{x}=l_{y}=0$ components is $99.15 \%$, in particular the $K=0$ component contribution is $94.4 \%$ and the $K=2, l_{x}=l_{y}=0$ component contribution is $3.3 \%$. This result agrees well with the results of other authors who used the three-body cluster model of ${ }^{11} \mathrm{Li}$ and the two-body interactions of the same type. In particular, the contribution of the $l_{x}=l_{y}=0$ components was estimated in Ref. [30] as 99\%. According to Ref. [33], the weight of the dominant $K=0, l_{x}=l_{y}=0$ wave function component is $95.3 \%$, and the contribution of this component together with the next $K=2, l_{x}=l_{y}=0$ component is $98.4 \%$. The dominant components of the ${ }^{11} \mathrm{Li}$ ground state wave function in the oscillator representation, $\left\langle\kappa, K=0, l_{x}=0, l_{y}=0: 00 \mid \Psi\right\rangle$ and $\left\langle\kappa, K=2, l_{x}=0, l_{y}=0: 00 \mid \Psi\right\rangle$, are shown in Fig. 3 . It is seen from the figure that the low- $\kappa$ dominating oscillator components are well reproduced in the variational calculation. However as $\kappa$ approaches the truncation boundary $\kappa_{\Gamma}$, the variational calculation underestimates $\left\langle\kappa, K=0, l_{x}=0, l_{y}=0: 00 \mid \Psi\right\rangle$ and $\left\langle\kappa, K=2, l_{x}=0, l_{y}=0: 00 \mid \Psi\right\rangle$ essentially.

A very interesting problem is the problem of low-energy electromagnetic transitions in ${ }^{11} \mathrm{Li}$ and other neutron-rich loosely-bound nuclei. It is supposed that the $E 1$ transition strength is strongly enhanced in such nuclei at small enough energies (the so-called soft dipole mode). We calculate the reduced E1 transi- 
tion probability

$$
\frac{d \mathcal{B}(E 1)}{d E}=\frac{1}{2 J_{i}+1} \sum_{J_{f}}\left|\left\langle J_{f} \| \mathscr{M}(E 1)|| J_{i}\right\rangle\right|^{2}
$$

where

$$
\mathscr{M}(E 1 \mu)=-\frac{Z e n}{A} \sqrt{\frac{\hbar}{\omega} \frac{2 m+M_{c}}{2 m M_{c}}} y Y_{1 \mu}(\hat{\boldsymbol{y}}),
$$

$\boldsymbol{y}$ is the Jacobi coordinate proportional to the distance between the ${ }^{9} \mathrm{Li}$ cluster and the center of mass of two valent neutrons, $m$ is the nucleon mass and $M_{c}$ is the ${ }^{9} \mathrm{Li}$ cluster mass, $e$ is the electron charge, the number of protons in ${ }^{11} \mathrm{Li}$ $Z=3$, the number of valent neutrons $n=2, J_{i}=3 / 2$ and $J_{f}$ are the total angular momenta of the ground (initial) and final states, respectively. We note here that within our three-body cluster model of ${ }^{11} \mathrm{Li}$ we calculate only the so-called cluster E1 transition strength associated with the relative motion of two neutrons and the ${ }^{9} \mathrm{Li}$ cluster; the total $E 1$ transition strength includes additionally excitations of nucleons forming the ${ }^{9} \mathrm{Li}$ cluster that manifest itself at higher energies. Since at low energies the only open decay channel is the three-body one, ${ }^{11} \mathrm{Li} \rightarrow{ }^{9} \mathrm{Li}+n+n$, the democratic decay approximation is well justified for the calculation of the final state wave function.

The results of the $\frac{d \mathcal{B}(E 1)}{d E}$ calculations are presented in Fig. 4 . It is interesting to compare the results obtained in the following approximations:

$\mathrm{VV}$ - the variational calculation of both the ground and final states;

VJ - the variational calculation of the ground state and the $J$-matrix calculation of the final three-body continuum state; and

JJ - the $J$-matrix calculation of both the ground and final three-body continuum states.

In the VV approximation, we obtain a discrete spectrum of excited states and cannot calculate the final state wave function at an arbitrary given positive energy. As a result, the $E 1$ transition strength differs from zero at the energies belonging to the discrete spectrum of final states and $\frac{d \mathcal{B}(E 1)}{d E}$ has a form of a number of $\delta$-function peaks shown in the figure by vertical lines with a cross at the end. The first peak at the excitation energy of approximately $2 \mathrm{MeV}$ exhausts nearly $80 \%$ of the cluster energy-weighted sum rule [38]

$$
\mathcal{S}_{E 1}^{\text {clust }}=\int_{0}^{\infty}\left(E_{f}-E_{0}\right) \frac{d \mathcal{B}(E 1)}{d E} d E_{f}=\frac{9}{4 \pi} \frac{\hbar^{2} e^{2}}{2 m} \frac{n Z^{2}}{A(A-n)},
$$

where $E_{0}$ is the ground state energy. This is clearly seen from Fig. 5 where we 


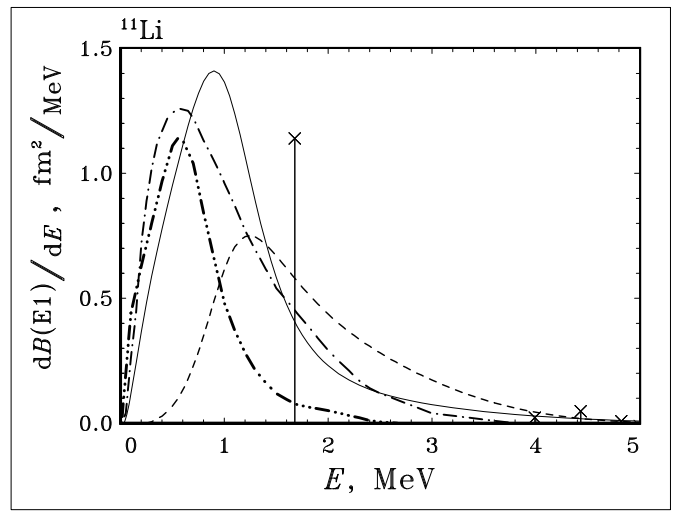

Fig. 4. Reduced $E 1$ transition probability $\frac{d \mathcal{B}(E 1)}{d E}$ in ${ }^{11} \mathrm{Li}$. Vertical lines with cross at the end, dashed and solid lines were obtained in the VV, VJ and JJ approximations, respectively, with $\hbar \omega=6.5 \mathrm{MeV}$ and $\tilde{N}=20$ for the ground state and $\tilde{N}=21$ for the final state. Dash-dot and dash-dot-dot lines are the calculations of Ref. [39] and the parameterizations of experimental data of Ref. [40], respectively.

present the plot of the function

$$
\mathcal{S}_{E 1}(E)=\frac{1}{\mathcal{S}_{E 1}^{\text {clust }}} \int_{0}^{E}\left(E_{f}-E_{0}\right) \frac{d \mathcal{B}(E 1)}{d E} d E_{f}
$$

The $2 \mathrm{MeV}$ peak should be related with the soft dipole mode in ${ }^{11} \mathrm{Li}$ in the VV approach. The energy of the soft dipole mode obtained in the VV approximation differs essentially from the one of the experimental $E 1$ strength low-energy maximum.

In the VJ approximation, the final energy wave function can be calculated at any given positive energy. Instead of sharp $\delta$-peaks, we have a smooth $\frac{d \mathcal{B}(E 1)}{d E}$ curve with a maximum shifted to a lower energy that is closer to the energy of the experimental $E 1$ strength maximum. This is clearly the result of allowing for the continuum spectrum effects in the final state wave function. However the external 'asymptotic' part of the model space with $2 \kappa+K>\tilde{N}$ is not allowed for in the ground state wave function and hence the final state wave function components with $2 \kappa+K>\tilde{N}+2$ do not contribute to $\frac{d \mathcal{B}(E 1)}{d E}$.

The contributions to $\frac{d \mathcal{B}(E 1)}{d E}$ of the external asymptotic part of the model space of both the ground state and the final state wave functions, are com- 


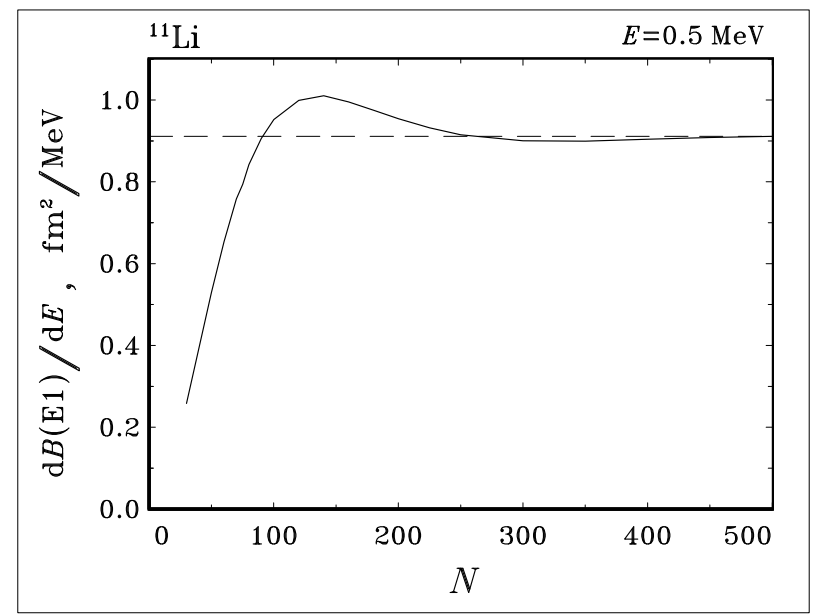

Fig. 6. The $\frac{d \mathcal{B}(E 1)}{d E}$ convergence with $N$ at the energy $E=0.5 \mathrm{MeV}$ where all components with the number of oscillator quanta $2 \kappa+K \leq N$ are allowed for in the JJ calculations with $\hbar \omega=6.5 \mathrm{MeV}$ and $\tilde{N}=20$ for the ground state and $\tilde{N}=21$ for the final state.

pletely accounted for in the JJ approximation. These contributions are seen from Figs. 4 and 5 to be of great importance. They shift the $\frac{d \mathcal{B}(E 1)}{d E}$ maximum to lower energy and change the shape of the $E 1$ strength function. We note here that due to the slow decrease in the asymptotic region of the wave function of the loosely-bound state, it is needed to allow for a very large number of components in the asymptotic part of the model space in the $E 1$ strength calculations. To calculate $\frac{d \mathcal{B}(E 1)}{d E}$ in the low-energy region with high accuracy, we should allow for all components with the number of oscillator quanta $2 \kappa+K \leq N$ where $N$ is of the order of 1000 . The convergence of $\frac{d \mathcal{B}(E 1)}{d E}$ with $N$ at the energy $E=0.5 \mathrm{MeV}$, is shown in Fig. 6. We note here that the $N \hbar \omega$ oscillator function has the classical turning point at

$$
\sqrt{\frac{\hbar}{m \omega}} \rho \sqrt{\frac{2 N \hbar}{m \omega}}
$$

Therefore supposing, say, $N=500$, we allow for the distances up to $\sqrt{\frac{\hbar}{m \omega}} \rho \approx$ $80 \mathrm{fm}$ in the $\frac{d \mathcal{B}(E 1)}{d E}$ calculation with $\hbar \omega=6.5 \mathrm{MeV}$.

\section{Two-neutron halo in ${ }^{6} \mathrm{He}$ nucleus}

The ${ }^{6} \mathrm{He}$ nucleus is studied in the three-body cluster model ${ }^{6} \mathrm{He}=\alpha+n+n$. The ${ }^{6} \mathrm{He}$ two-neutron separation energy is $0.976 \mathrm{MeV}$ [41] that is much less 
than the excitation energy of the $\alpha$ particle lowest excited state and the $\alpha$ particle disintegration threshold. As it was already noted, ${ }^{6} \mathrm{He}$ is a Borromean nuclei, i. e. none of the two-body subsystems $\alpha+n$ and $n+n$ has a bound state.

Generally our approach in the ${ }^{6} \mathrm{He}$ case is very close to the one used in the ${ }^{11} \mathrm{Li}$ studies discussed above. However, contrary to the $n-{ }^{11} \mathrm{Li}$ interaction, the $n-\alpha$ interaction is well-known. Therefore it is reasonable to use a more microscopically justified approach based on available more realistic $n$-cluster and $n-n$ potentials. In particular, it is reasonable to explicitly allow for the spin variables in our model. We couple the spins of two valent neutrons into the total spin $S=0,1$ (the $\alpha$ particle has a zero spin), the orbital angular momenta $l_{x}$ and $l_{y}$ are coupled to the total orbital angular momentum $L$, and $L$ and $S$ are coupled to the total angular momentum $J$. Hence the channel index $\Gamma=\left\{K, l_{x}, l_{y}, L, S, J\right\}$, and we introduce the following generalization of the basis functions (42):

$$
\left|\kappa K l_{x} l_{y}(L)(S): J M\right\rangle=\sum_{M_{L}, M_{S}}\left(L M_{L} S M_{S} \mid J M\right)\left|\kappa K l_{x} l_{y}: L M_{L}\right\rangle\left|S M_{S}\right\rangle
$$

the ${ }^{6} \mathrm{He}$ wave function is given by the general formula (12). In Eq. (53), $\left|S M_{S}\right\rangle$ is the spin component of the wave function and $\left|\kappa K l_{x} l_{y}: L M_{L}\right\rangle$ is given by Eqs. (42)-(43). We note here that allowing for the triplet $(S=1)$ spin states, we enlarge essentially the number of basis functions with any given number of oscillator quanta $N=2 \kappa+K$. Hence for any given $\tilde{N}$, we have the truncated Hamiltonian matrix of a much larger rank, and we are able to perform calculations with smaller $\tilde{N}$ than in the ${ }^{11} \mathrm{Li}$ case.

It would be very interesting to perform the ${ }^{6} \mathrm{He}$ studies based on modern socalled realistic $N N$ potentials derived from the meson exchange theory and perfectly describing $N N$ scattering data and deuteron observables. However the oscillator basis matrices of such potentials are extremely large and cannot be handled in calculations of lightest nuclei; the realistic potential matrix elements decrease slowly with the number of oscillator quanta $N$ and truncation of the Hamiltonian matrix results in the slow convergence of the results. In practical applications usually effective interactions are used in calculations. Microscopic ab initio approaches (see, e. g., [42]) involve realistic effective interactions and effective operators derived from the realistic $N N$ potentials. Unfortunately the $J$-matrix formalism (and other formalisms allowing for the continuum spectrum effects) is not developed still for the case of ab initio models based on realistic effective $N N$ interactions. In this contribution, we are interested in the continuum spectrum effects and $S$-matrix pole corrections to the binding energy, and so we use phenomenological effective potentials for $n-n$ and $n-\alpha$ interactions. A large number of phenomenological effective $n-n$ and $n-\alpha$ potentials is available. We perform calculations with various $n-n$ and $n-\alpha$ potentials since it is not clear from the very beginning what is the 
best choice of these potentials; we would like also to compare our results with the results of other authors who used different combinations of these interactions.

The following effective $n-n$ potentials were employed in our studies.

The Gaussian potential (38) with $V_{0}=30.93 \mathrm{MeV}$ and $R=1.82 \mathrm{fm}$ in the singlet $(S=0)$ state and $V_{0}=60.9 \mathrm{MeV}$ and $R=1.65 \mathrm{fm}$ in the triplet $(S=1)$ state suggested in Ref. [43], is hereafter refered to as Gs. The potential was fitted to the $s$ wave nucleon-nucleon phase shifts only; the interaction between the neutrons in the states with relative orbital angular momentum $l_{x}>0$, is neglected.

We also make use of a bit different Gaussian potential suggested in Refs. [44,45] with $V_{0}=31.00 \mathrm{MeV}$ and $R=1.8 \mathrm{fm}$ in the singlet state and $V_{0}=71.09 \mathrm{MeV}$ and $R=1.4984 \mathrm{fm}$ in the triplet state. The singlet component of this potential was employed in our ${ }^{11} \mathrm{Li}$ studies. We shall refer to this potential as $\mathrm{G}$.

A more realistic Minnesota $n-n$ potential of Ref. [46] will be refered to as MN. This Gaussian potential includes central, spin-orbit and tensor components.

The lowest single particle $0 s_{1 / 2}$ is occupied in the $\alpha$ particle. There are two conventional approaches to the problem of the Pauli forbidden $0 s_{1 / 2}$ state in the $n+\alpha$ system. The first approach is to add a phenomenological repulsive term to the $s$ wave component of the $n-\alpha$ potential. This phenomenological repulsion excludes the Pauli forbidden state in the $n+\alpha$ system and is supposed to simulate the Pauli principle effects in more complicated cluster systems. This idea was utilized in the SBB $n-\alpha$ potential suggested in Ref. [47]. This is an $l$-dependent Gaussian potential that includes the spin-orbit component.

Another approach is to use a deep attractive $n-\alpha$ potentials that support the Pauli forbidden $0 s_{1 / 2}$ state in the $n+\alpha$ system. The Pauli forbidden states should be excluded in the three-body cluster system. The conventional method is to supplement the deep attractive $n-\alpha$ potential by the projecting pseudo potential (see, e. g. [48])

$$
\lambda\left|0 s_{1 / 2}\right\rangle\left\langle 0 s_{1 / 2}\right| .
$$

If the parameter $\lambda$ is positive and large enough, the projector (54) pushes the Pauli forbidden states to very large energies and cleans up the ground and low-lying states from the Pauli forbidden admixtures. The eigenfunction of the Pauli forbidden state supported by the deep attractive $n-\alpha$ potential should be used as $\left|0 s_{1 / 2}\right\rangle$ (the so-called eigen-projector [48]); in this case the pseudo potential (54) does not affect the description of the scattering data provided by the initial deep attractive $n-\alpha$ potential.

The deep attractive Woods-Saxon $n-\alpha$ potential suggested in Ref. [49] will be hereafter refered to as WS. We use the WS potential parameters suggested 
in Ref. [36] where the radius of the potential was increased in order to fit the ${ }^{6}$ He binding energy.

We use also deep attractive Majorana splitting potential suggested in Ref. [48] (hereafter refered to as MS) and deep attractive $l$-dependent Gaussian potential proposed in Ref. [26] (hereafter refered to as GP). These potentials improve the description of scattering data in high $(l>1)$ partial waves.

The convergence patterns in the ${ }^{6} \mathrm{He}$ case are very similar to the ones discussed in the ${ }^{11} \mathrm{Li}$ case. As a typical example, we present the results obtained in the Gs + SBB potential model. The ${ }^{6} \mathrm{He}$ ground state energy dependence on the oscillator parameter $\hbar \omega$ for a number of truncation boundary $\tilde{N}$ values, is depicted in Fig. 7. The $\hbar \omega$ ground state energy dependence is seen to be of the same type as the one in the ${ }^{11} \mathrm{Li}$ case shown in Fig. 1 . The ${ }^{6} \mathrm{He}$ ground state energy and rms radius convergence with $\tilde{N}$ is presented in Fig. 8 for two $\hbar \omega$ values. As in the ${ }^{11} \mathrm{Li}$ case (see Fig. 2), there is the staggering of the $J$-matrix binding energy and rms radius with $\tilde{N}$ for $\hbar \omega=10 \mathrm{MeV}$ that is close to the value providing the best convergence of the variational calculation, and there is the variational-type $\tilde{N}$ dependence of these observables for larger $\hbar \omega$ values. It is interesting that all calculations shown on the right panel of Fig. 7 , result in nearly the same binding energy at $\hbar \omega \approx 15 \mathrm{MeV}$; this $\hbar \omega$ value is clearly the best convergence value for the $J$-matrix calculation and it differs essentially from the best convergence $\hbar \omega$ value for the variational calculation corresponding to the minima of the curves on the left panel of Fig. 7.

The results of our calculations of the ${ }^{6} \mathrm{He}$ two-neutron separation energy and rms matter radius, are summarized in Table 2. Since the calculations using different potential models were performed with slightly different $\hbar \omega$ values, we list these values in the Table. If for a given potential model the theoretical predictions of other authors using other approximations within the three-body cluster model are available, they are presented in the corresponding rows of the Table, too. The theoretical predictions within the three-body cluster model with other potential models and the experimental data, are presented in the Table in additional rows.

The $J$-matrix approach is seen from the Table to improve the variational results for both the binding energy and the rms radius in the case of any potential model. The improvement is generally more essential in the case of smaller binding energy (it is also seen comparing the ${ }^{6} \mathrm{He}$ results in Table 2 with the ${ }^{11} \mathrm{Li}$ results in Table 1 ). The $J$-matrix approach is also seen to have a faster convergence than the variational one, and again the improvement of the convergence rate is more essential in the smaller binding energy case. Our results are in good correspondence with the available results of other authors who used the same potential models. 

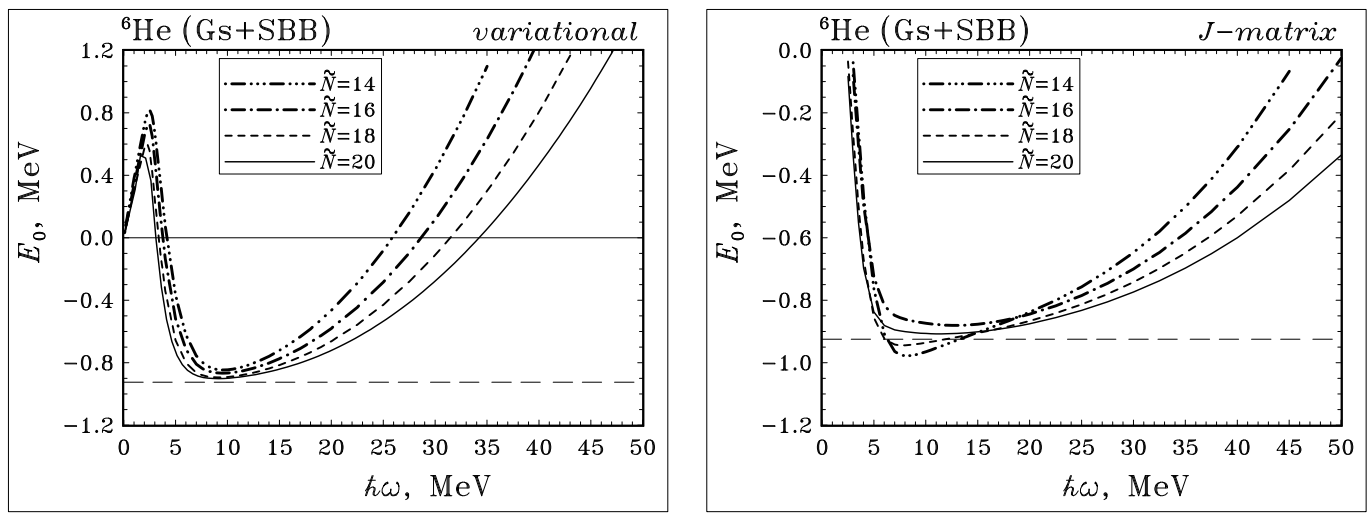

Fig. 7. The ${ }^{6} \mathrm{He}$ ground state energy in the Gs+SBB potential model vs the oscillator basis parameter $\hbar \omega$ in the variational (left panel) and $J$-matrix (right panel) calculations for different values of the truncation boundary $\tilde{N}$. The horizontal dashed line depicts the convergence limit for the ground state energy.
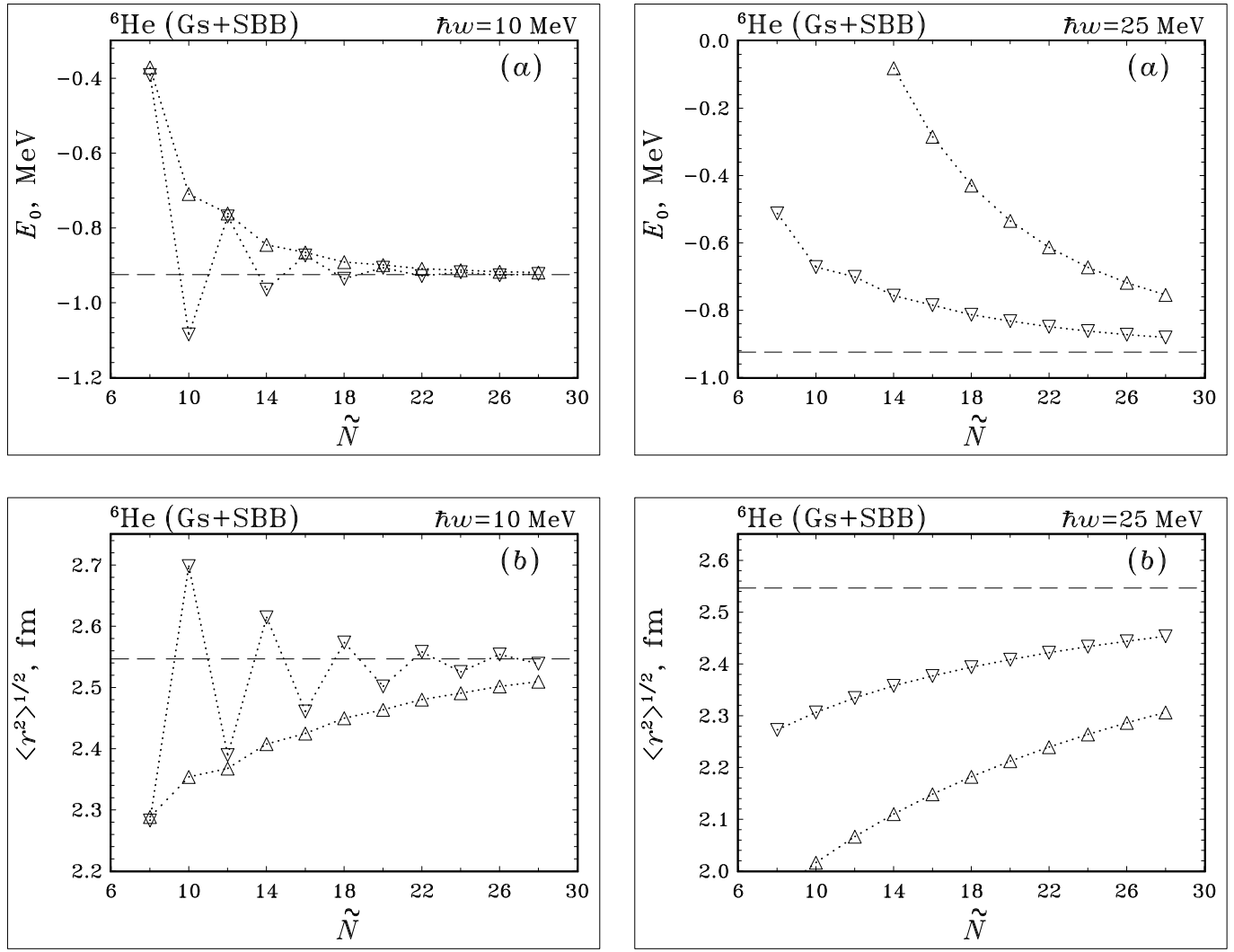

Fig. 8. Convergence with $\tilde{N}$ of the ${ }^{6} \mathrm{He}$ ground state energy (a) and rms matter radius (b) in the Gs $+\mathrm{SBB}$ potential model for $\hbar \omega=10 \mathrm{MeV}$ (left panel) and $\hbar \omega=25 \mathrm{MeV}$ (right panel). Variational and $J$-matrix results are shown by triangles up and triangles down, respectively. The convergence limits are shown by the dashed line. 
Table 2

The ${ }^{6} \mathrm{He}$ two-neutron separation energy and rms matter radius obtained in various potential models and available three-body cluster model results of other authors.

\begin{tabular}{|c|c|c|c|c|c|c|}
\hline \multirow{2}{*}{ Potential model } & \multirow{2}{*}{$\hbar \omega, \mathrm{MeV}$} & \multirow{2}{*}{ Approximation } & \multicolumn{2}{|c|}{$E(2 n), \mathrm{MeV}$} & \multicolumn{2}{|c|}{$\left\langle r^{2}\right\rangle^{1 / 2}, \mathrm{fm}$} \\
\hline & & & $\tilde{N}=26$ & $\tilde{N}=28$ & $\tilde{N}=26$ & $\tilde{N}=28$ \\
\hline \multirow{3}{*}{$\mathrm{Gs}+\mathrm{SBB}$} & \multirow{2}{*}{10.00} & variational & 0.917 & 0.919 & 2.502 & 2.510 \\
\hline & & $J$-matrix & 0.927 & 0.923 & 2.554 & 2.539 \\
\hline & \multicolumn{2}{|c|}{ Ref. [47] } & \multicolumn{2}{|c|}{ "correct asymptotic value" } & & \\
\hline \multirow{3}{*}{$\mathrm{Gs}+\mathrm{WS}$} & \multirow{2}{*}{11.90} & variational & 0.993 & 0.997 & 2.394 & 2.404 \\
\hline & & $J$-matrix & 1.003 & 1.008 & 2.444 & 2.461 \\
\hline & \multicolumn{2}{|c|}{ Ref. [36] } & \multicolumn{2}{|c|}{1.00} & \multicolumn{2}{|c|}{2.44} \\
\hline \multirow{2}{*}{$\mathrm{G}+\mathrm{GP}$} & \multirow{2}{*}{11.35} & variational & 0.875 & 0.878 & 2.386 & 2.393 \\
\hline & & $J$-matrix & 0.889 & 0.883 & 2.451 & 2.428 \\
\hline \multirow{2}{*}{$\mathrm{MN}+\mathrm{GP}$} & \multirow{2}{*}{10.75} & variational & 0.494 & 0.509 & 2.477 & 2.484 \\
\hline & & $J$-matrix & 0.515 & 0.516 & 2.588 & 2.547 \\
\hline \multirow{2}{*}{$\mathrm{MN}+\mathrm{MS}$} & \multirow{2}{*}{13.00} & variational & 0.656 & 0.672 & 2.382 & 2.389 \\
\hline & & $J$-matrix & 0.684 & 0.681 & 2.482 & 2.445 \\
\hline \multicolumn{3}{|c|}{ other potential models } & \multicolumn{2}{|c|}{$\begin{array}{lll}\text { Ref. [48]: } 0.305 ; & \text { Ref. [50]: 1.00; } \\
\text { Ref. [51]: 0.784; } & \text { Ref. [52]: } 0.696\end{array}$} & \multicolumn{2}{|c|}{ Ref. [50]: 2.50} \\
\hline \multicolumn{3}{|c|}{ experimental data } & \multicolumn{2}{|c|}{ Ref. [41]: 0.976} & \multicolumn{2}{|c|}{$\begin{array}{l}\text { Ref. [53]: } 2.33 \pm 0.04 \\
\text { Ref. [54]: } 2.48 \pm 0.03 \\
\text { Ref. [55]: } 2.57 \pm 0.10\end{array}$} \\
\hline
\end{tabular}


The structure of the ${ }^{6} \mathrm{He}$ ground state wave function is presented in Table 3. We note here that within the three-body cluster model ${ }^{6} \mathrm{He}=\alpha+n+n$, the $0^{+}$ground state can be obtained by the coupling of the Jacobi orbital momenta $l_{x}$ and $l_{y}$ to the total orbital angular momentum $L$ and subsequent coupling of $L$ with the total neutron spin $S$ into the total angular momentum $J=0$, if only $L=S$ and $l_{x}=l_{y}$. We arrange the dominant components in a different manner. In the Table 3 (a) we show the total weights of all components with given $S=L$ and $l_{x}=l_{y}$, i. e. we sum the contributions of the components with different $K$ and $N=2 \kappa+K$ values for the given $S=L$ and $l_{x}=l_{y}$ values. In the Table $3(\mathrm{~b})$ we collect the total weights of all components with given $S=L$ and hypermomentum $K$ summing the contributions with different $l_{x}=l_{y}$ values. It is also interesting to calculate the shell model type component weights which are characterized by the orbital momenta $l_{n_{1} \alpha}$ and $l_{n_{2} \alpha}$ of individual neutron motion relatively to the $\alpha$ core. The unitary transformation from the hyperspherical basis (53) to the shell model basis can be found in textbooks (see, e. g., $[59,60]$ ). The ${ }^{6} \mathrm{He} 0^{+}$ground state wave function can be arranged if only $l_{n_{1} \alpha}=l_{n_{2} \alpha}$. The shell model-type component weights are listed in the Table 3 (c).

It is seen from Table 3 that different potential models result in the wave functions of nearly the same structure. For example, the component weights in different arrangements obtained with SBB potential simulating the Pauli principle by repulsive terms and with WS potential when the Pauli forbidden states are explicitly projected out, are nearly the same. Our results for component weights are in good correspondence with the results of other authors who used other approaches to the three-body cluster model and other potential models. Therefore the uncertainties of the ${ }^{6} \mathrm{He}$ wave function structure due to the uncertainties of the two-body interactions, are very small. The most essential (however not very large) difference between the dominant component weights obtained in different approaches is the difference between the results of Ref. [48] where the RSC + SBB* potential model was employed and our results and the results of Ref. $[47,16]$ obtained with different potential models. We note here that the ${ }^{6} \mathrm{He}$ binding energy obtained in Ref. [48] is $0.3046 \mathrm{MeV}$ only, i. e. it was essentially underestimated. Most probably this is indication that the $\mathrm{RSC}+\mathrm{SBB}^{*}$ potential model is not adequate for the description of ${ }^{6} \mathrm{He}$ in the cluster model and hence it is not surprising that the component weights of Ref. [48] differ from the ones obtained with other potentials.

From the naive shell model considerations it follows that two valent neurons should occupy $p$ states in the ${ }^{6} \mathrm{He}$ nucleus. We see from the Table $3(\mathrm{c})$ that this situation is really utilized in the dominant component of the ${ }^{6} \mathrm{He}$ wave function. The same naive shell model considerations bring us to the $K=2$ component dominance, and again we see from the Table $3(\mathrm{~b})$ that this is really the case. However from these naive considerations it looks strange that the dominant component corresponds to the Jacobi orbital momenta $l_{x}=l_{y}=0$ 
Table 3

Dominant component weights in the ${ }^{6} \mathrm{He} 0^{+}$ground state wave function obtained with various potential models in the $J$-matrix approximation with $\tilde{N}=28$ (the respective $\hbar \omega$ values can be found in Table 2) and the results of other authors in the three-body cluster model.

(a) Total contribution of the components with given $S=L$ and $L_{x}=l_{y}$ (summation over possible $K$ and $N$ values)

\begin{tabular}{|c|c|c|c|c|c|c|c|c|}
\hline \multicolumn{2}{|c|}{ State } & \multicolumn{7}{|c|}{ Weight, \% } \\
\hline \multirow[b]{2}{*}{$S=I$} & \multirow[b]{2}{*}{$l_{x}=l_{y}$} & \multicolumn{4}{|c|}{ present work } & \multicolumn{2}{|c|}{ Ref. $[47,16]$} & \multirow{2}{*}{$\begin{array}{c}\text { Ref. }[48] \\
\text { RSC }^{2}+\text { SBB }^{* 3}\end{array}$} \\
\hline & & $\mathrm{Gs}+\mathrm{SBB}$ & $\mathrm{Gs}+\mathrm{WS}$ & $\mathrm{G}+\mathrm{GP}$ & $\mathrm{MN}+\mathrm{MS}$ & $\mathrm{Gs}+\mathrm{SBB}$ & $\mathrm{GPT}^{1}+\mathrm{SBB}$ & \\
\hline \multirow{3}{*}{0} & 0 & 82.1 & 83.6 & 80.2 & 76.6 & 83.13 & 82.87 & 88.908 \\
\hline & 2 & 2.46 & 1.90 & 1.64 & 2.07 & 1.77 & 2.31 & 1.035 \\
\hline & 4 & 0.18 & 0.14 & 0.11 & 0.15 & 0.02 & 0.58 & \\
\hline \multirow{3}{*}{1} & 1 & 14.3 & 13.4 & 17.6 & 20.1 & 14.54 & 13.96 & 9.692 \\
\hline & 3 & 0.85 & 0.83 & 0.66 & 0.89 & 0.55 & 0.69 & 0.366 \\
\hline & 5 & 0.09 & 0.09 & 0.06 & 0.09 & & 0.06 & \\
\hline
\end{tabular}

${ }^{1}$ Gogny-Pires-de Tourreil $n-n$ potential [56].

${ }^{2}$ Reid soft-core $n-n$ potential [57].

${ }^{3} \mathrm{SBB} n-\alpha$ potential with modified parameters (see Ref. [48]). 
Table 3

Prolongation

(b) Total contribution of the components with given $K$ and $S=L$ (summation over possible $L_{x}=l_{y}$ values)

\begin{tabular}{|cc|cccc|cc|}
\hline \multicolumn{2}{|c|}{ State } & \multicolumn{5}{|c|}{ Weight, \% } & \multicolumn{2}{c|}{ Ref. [47,16] } \\
\hline \multirow{3}{*}{$S=L$} & & \multicolumn{5}{|c|}{ present work } \\
& $K$ & Gs + SBB & Gs + WS & G + GP & MN + MS & Gs + SBB & GPT + SBB \\
\hline & 0 & 4.28 & 4.06 & 3.99 & 3.93 & 4.41 & 4.68 \\
& 2 & 77.2 & 78.9 & 75.5 & 72.4 & 78.93 & 78.10 \\
& 4 & 0.59 & 0.19 & 0.13 & 0.16 & 0.53 & 0.64 \\
& 6 & 1.60 & 1.61 & 1.40 & 1.73 & 1.16 & 1.43 \\
& 8 & 0.67 & 0.51 & 0.43 & 0.41 & 0.39 & \\
\hline & 2 & 14.2 & 13.2 & 17.5 & 20.0 & 13.91 & 13.41 \\
& 4 & 0.12 & 0.15 & 0.05 & 0.06 & 0.12 & 0.15 \\
& 6 & 0.82 & 0.80 & 0.63 & 0.85 & 0.53 & 0.67 \\
& 6 & 0.05 & 0.04 & 0.04 & 0.06 & 0.02 & \\
\hline
\end{tabular}


Table 3

Prolongation

(c) Total contribution of the components with given shell model-like orbital angular momenta $l_{n_{1} \alpha}=l_{n_{2} \alpha}$

\begin{tabular}{|c|cccc|c|}
\hline State & \multicolumn{5}{|c|}{ Weight, \% } \\
\hline \multirow{2}{*}{$l_{n_{1} \alpha}=l_{n_{2} \alpha}$} & \multicolumn{5}{|c|}{ present work } \\
\hline$s$ & 8.27 & 7.60 & 7.35 & 7.26 & 7.7 \\
$p$ & 90.4 & 90.9 & 91.5 & 91.6 & 91.0 \\
$d$ & 0.57 & 0.64 & 0.43 & 0.44 & GPT $+\mathrm{WS}+\mathrm{V}_{3} 4$ \\
$f$ & 0.57 & 0.54 & 0.47 & 0.41 & \\
$g$ & 0.07 & 0.07 & 0.07 & 0.05 & \\
\hline
\end{tabular}

${ }^{4}$ Three-body nn $\alpha$ potential, see Ref. [58]. 


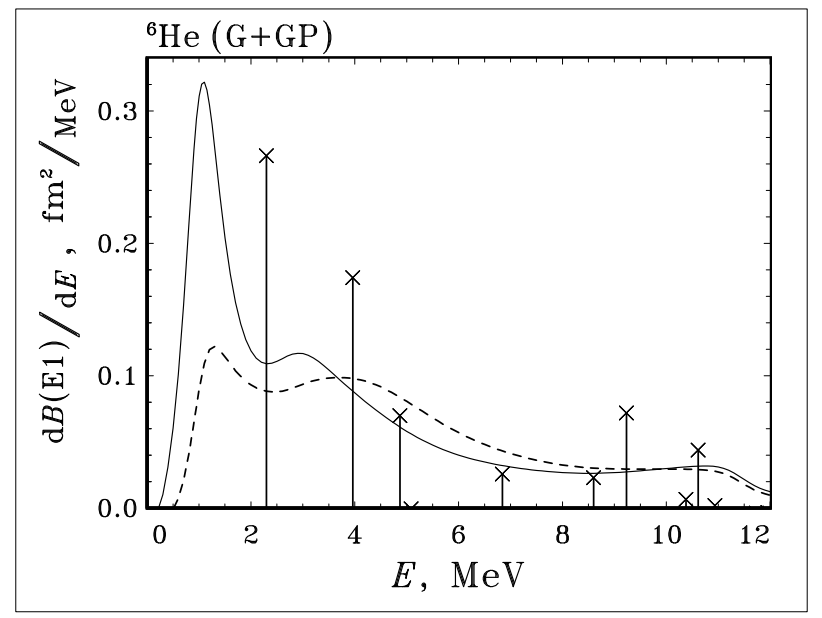

Fig. 9. Reduced $E 1$ transition probability $\frac{d \mathcal{B}(E 1)}{d E}$ in ${ }^{6} \mathrm{He}$ obtained in the G + GP potential model. Vertical lines with cross at the end, dashed and solid lines were obtained in the VV, VJ and JJ approximations, respectively, with $\hbar \omega=11.35 \mathrm{MeV}$ and $\tilde{N}=22$ for the ground state and $\tilde{N}=23$ for the final state.

[see the Table 3 (a)]. Nevertheless there is, of course, no contradiction between the results presented in the Table 3 (a) and the Table 3 (c), they are obtained by the summation over different quantum numbers of the same ${ }^{6} \mathrm{He}$ ground state wave functions.

An example of the reduced (cluster) E1 transition probability calculations in ${ }^{6} \mathrm{He}$ is shown in Fig. 9 where the results obtained with $\mathrm{G}+\mathrm{GP}$ potential model in the VV, VJ and JJ approximations are presented. In the ${ }^{6} \mathrm{He}$ case, the transition strength is distributed in the VV approximation over a number of strong $\delta$-peaks. Therefore the shape of the reduced probability $\frac{d \mathcal{B}(E 1)}{d E}$ appears to be more complicated than in the ${ }^{11} \mathrm{Li}$ case when the effects of continuum are allowed for in the VJ and JJ approximations. As in the ${ }^{11} \mathrm{Li}$ case, we see that the external asymptotic part of the model space allowed for in the JJ approximation only, provides a very significant contribution to the electromagnetic transition probabilities.

The convergence of the reduced $E 1$ transition probability calculations in the JJ approximation is illustrated by Fig. 10 where we show the results obtained with different truncation boundaries in the ground state calculations $\tilde{N}_{\text {g.s. }}$, the truncation boundary in the final state calculations $\tilde{N}_{\text {f.s. }}=\tilde{N}_{\text {g.s. }}+1$. The staggering of the $\frac{d \mathcal{B}(E 1)}{d E}$ shape as $\tilde{N}_{\text {g.s. }}$ increases, is clearly seen in the figure. For example, the weakest transition strength is obtained with $\tilde{N}_{\text {g.s. }}=12$ and $\tilde{N}_{\text {f.s. }}=13$ shown by the dotted line in the figure; the strongest transition strength is obtained with $\tilde{N}_{\text {g.s. }}=14$ and $\tilde{N}_{\text {f.s. }}=15$ shown by the dash-dot line; the transition strength obtained with $\tilde{N}_{\text {g.s. }}=16$ and $\tilde{N}_{\text {f.s. }}=17$ is stronger than the one obtained with $\tilde{N}_{\text {g.s. }}=12$ and $\tilde{N}_{\text {f.s. }}=13$ but weaker than all the 


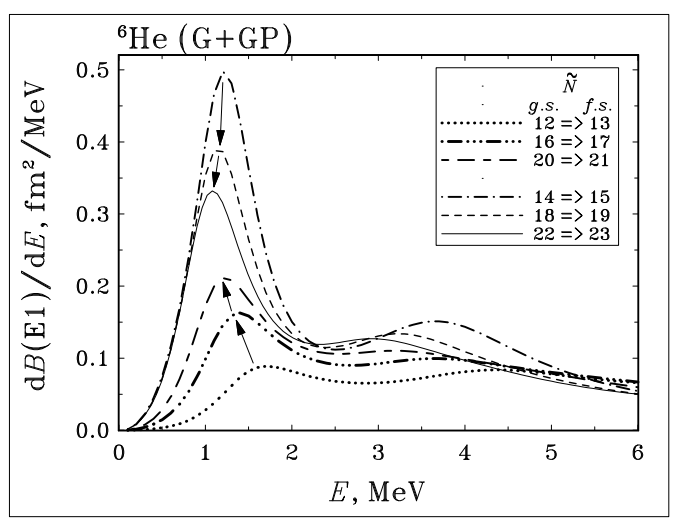

Fig. 10. Convergence of the $\frac{d \mathcal{B}(E 1)}{d E}$ calculations in ${ }^{6} \mathrm{He}$ with the truncation parameter $\tilde{N}_{\text {g.s. }}$ used in the ground state calculations (the final state truncation parameter $\left.\tilde{N}_{\text {f.s. }}=\tilde{N}_{\text {g.s. }}+1\right)$. The results are obtained in the JJ approximation with the $\mathrm{G}+\mathrm{GP}$ potential model and $\hbar \omega=11.35 \mathrm{MeV}$. Arrows show the changes of $\frac{d \mathcal{B}(E 1)}{d E}$ when $\tilde{N}_{\text {g.s. }}$ is increased by 4 .

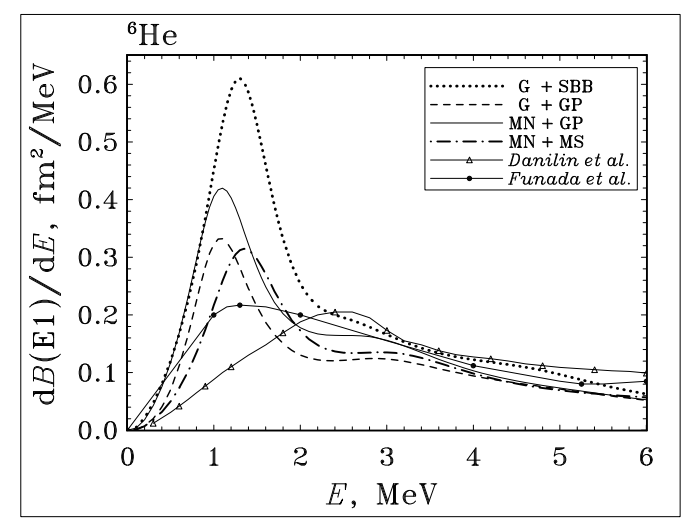

Fig. 11. Reduced E1 transition probability $\frac{d \mathcal{B}(E 1)}{d E}$ in ${ }^{6} \mathrm{He}$ obtained with various potential models in the JJ approximation with the ground state truncation parameter $\tilde{N}_{\text {g.s. }}=22$ and the final state truncation parameter $\tilde{N}_{\text {f.s. }}=23$ (the corresponding $\hbar \omega$ values can be found in Table 2) in comparison with the results of Funada et al. [61] and Danilin et al. [62].

rest results presented in the figure, etc. However selecting the results obtained with even $\tilde{N}_{\text {g.s. }} / 2$ values only, we see that the $E 1$ transition strength increases monotonically with $\tilde{N}_{\text {g.s. }}$; selecting the results obtained with odd $\tilde{N}_{\text {g.s. }} / 2$ values only, we see the monotonic decrease of the $E 1$ transition strength with $\tilde{N}_{\text {g.s. }}$.

The comparison of the reduced $E 1$ transition probability results obtained with different potential models and calculations of other authors within the threebody cluster model, is shown in Fig. 11.

\section{Phase equivalent transformation with continuous parameters and three-body cluster system}

The results presented in Table 2 show that we obtain a very good approximation for the ${ }^{6} \mathrm{He}$ two-neutron separation energy. However the exact value of the ${ }^{6} \mathrm{He}$ binding energy is not reproduced with any potential model employed. We suggest to use a phase equivalent transformation of the two-body interaction to improve the description of the ${ }^{6} \mathrm{He}$ binding. If the phase equivalent transformation depends on a continuous parameter(s) than varying the parameter we can fit the ${ }^{6} \mathrm{He}$ binding energy to the phenomenological value. We suppose this approach to be interesting for various few-body applications. 
Various phase equivalent transformations have been discussed in literature. The local phase equivalent transformations (transforming a local potential into another local potential phase equivalent to the initial one) is well-known (see, e. g., Ref. [63]). In the inverse scattering theory, this transformation gives rise to the ambiguity of the potential restored from the scattering data. Recently this transformation was extended on the case of the multichannel scattering [64]. However the local phase equivalent transformation can be applied only to a two-body system that have at least one bound states and the number of continuous parameters of the transformation is equal to the number of the bound states in the system. Therefore this transformation cannot be applied to the $n+\alpha$ system that has no bound states.

Recently the so-called supersymmetry phase equivalent transformation (see, e. g., review [65]) become very popular. Using this transformation one can transform a potential with the Pauli forbidden state into exactly phase equivalent potential with additional repulsion simulating the Pauli effects. The effect of this transformation on the properties of three-body cluster systems was examined in a number of recent papers (see, e. g., Ref. [66]). The supersymmetry transformation was shown [64] to be a particular case of the local phase equivalent transformation. However the supersymmetry transformation does not have parameters and cannot be used for our purposes.

A phase equivalent transformation based on unitary transformation of the Hamiltonian, was suggested in Ref. [67]. This transformation has continuous parameters. This transformation have been used in many-body applications in Ref. [68]. However the authors of Ref. [68] used few particular cases of the transformation corresponding to particular parameter values and did not try to vary the parameters continuously.

We have developed recently [26] a phase equivalent transformation using the $J$-matrix formalism. Generally, our transformation is a particular case of the phase equivalent transformation of Ref. [67]. However we suppose that our phase equivalent transformation is general enough and very convenient for the use in various many-body applications utilizing any $L^{2}$ basis. We applied this transformation to the $N N$ interaction and studied the effect of the transformation on the properties ${ }^{3} \mathrm{H}$ and ${ }^{4} \mathrm{He}$ nuclei in Ref. [69]. In what follows, we discuss briefly the transformation and its application to the ${ }^{6} \mathrm{He}$ nucleus within the three-body cluster model.

We suppose that a two-body system is described by the Schrödinger equation

$$
H \Psi(r)=E \Psi(r),
$$

where the Hamiltonian $H=T+V, T$ and $V$ are kinetic and potential energy operators. Introducing a complete basis $\left\{\phi_{\kappa}(r)\right\}, \kappa=0,1,2, \ldots$ of $L^{2}$ functions $\phi_{\kappa}(r)$, we can expand the solutions of Eq. (55) in infinite series of basis 
functions $|\kappa\rangle \equiv \phi_{\kappa}(r)$ :

$$
\Psi(r)=\sum_{\kappa=0}^{\infty} C_{\kappa}|\kappa\rangle .
$$

The Schrödinger equation (55) takes the form of an infinite dimensional algebraic problem

$$
\sum_{\kappa^{\prime}=0}^{\infty}\left\langle\kappa|H| \kappa^{\prime}\right\rangle C_{\kappa^{\prime}}=E C_{\kappa},
$$

where $\left\langle\kappa|H| \kappa^{\prime}\right\rangle$ are the matrix elements of the infinite dimensional Hamiltonian matrix $[H]$.

Now we define a new matrix

$$
\left[H^{\prime}\right]=\left[U^{+}\right][H][U]
$$

with the help of the unitary matrix $[U]$ which is supposed to be of the form

$$
[U]=\left[U_{0}\right] \oplus[I]=\left(\begin{array}{c|c}
{\left[U_{0}\right]} & 0 \\
\hline 0 & {[I]}
\end{array}\right),
$$

where $[I]$ is the infinite dimensional unit matrix and $\left[U_{0}\right]$ is $N \times N$ unitary matrix. A new Hamiltonian $H^{\prime}$ is defined through its matrix $\left[H^{\prime}\right]$. It is supposed that $\left[H^{\prime}\right]$ is the matrix of the Hamiltonian $H^{\prime}$ in the original basis $\left\{\phi_{\kappa}(r)\right\}$.

Clearly the spectra of Hamiltonians $H$ and $H^{\prime}$ are identical. The difference between the eigenfunctions $\Psi^{\prime}(r)$ of the Hamiltonian $H^{\prime}$ and the eigenfunctions $\Psi(r)$ of the Hamiltonian $H$ corresponding to the same energy $E$, is a superposition of a finite number of square integrable functions:

$$
\Psi^{\prime}(r)=\Psi(r)+\sum_{\kappa=0}^{N-1} \Delta C_{\kappa} \phi_{\kappa}(r) .
$$

The superposition of a finite number of $L^{2}$ functions cannot affect the asymptotics of scattering wave functions. Since the scattering phase shifts and the $S$-matrix are defined through the asymptotic behavior of the wave functions, the phase shifts associated with the functions $\Psi(r)$ and $\Psi^{\prime}(r)$ are identical. In other words, the Hamiltonians $H$ and $H^{\prime}$ are phase equivalent.

Supposing that the Hamiltonian $H^{\prime}=T+V_{\mathrm{PET}}$, we introduce the potential

$$
V_{\mathrm{PET}}=V+\Delta V
$$

phase equivalent to the original potential $V$. The potential $V_{\text {PET }}$ is defined through its matrix

$$
\left[V_{\mathrm{PET}}\right]=[V]+[\Delta V]
$$


in the basis $\left\{\phi_{\kappa}(r)\right\}$, where

$$
[\Delta V]=\left[U^{+}\right][H][U]-[H]
$$

The net result of the above considerations is very simple. We introduce any complete $L^{2}$ basis $\left\{\phi_{n}(r)\right\}$ and calculate the Hamiltonian matrix in this basis. Next we introduce an arbitrary unitary transformation of the type (59) that affects a finite number of the basis functions. With the help of this transformation, we calculate the additional potential $\Delta V$ using Eq. (63) and obtain the phase equivalent interaction by means of Eq. (61). The additional potential $\Delta V$ is non-local, and hence the phase equivalent potential $V_{\text {PET }}$ is non-local, too. Therefore the suggested transformation is a non-local phase equivalent transformation: it brings us to a non-local interaction phase equivalent to the original one.

The suggested non-local phase equivalent transformation can be easily implemented in many-body calculations utilizing any $L^{2}$ basis. One just needs to add the two-body kinetic energy matrix to the two-body interaction matrix, unitary transform the obtained matrix and subtract the kinetic energy matrix from the result to obtain the matrix of the phase equivalent two-body interaction.

The non-local phase equivalent transformation can be easily explained and understood within the $J$-matrix formalism. In the $J$-matrix formalism, the $S$ matrix and the phase shifts are governed by the matrix elements $\left\langle\kappa_{\Gamma} \Gamma|\mathscr{P}| \kappa_{\Gamma^{\prime}}+1, \Gamma^{\prime}\right\rangle$ which are defined through eigenvalues $E_{\lambda}$ and the last component $\left\langle\kappa_{\Gamma} \Gamma \mid \lambda\right\rangle$ of the eigenvectors of the truncated Hamiltonian matrix [see Eqs. (30)-(31)]. If the truncated Hamiltonian matrix is larger than the non-trivial submatrix $\left[U_{0}\right]$ of the unitary matrix $[U]$, than neither $E_{\lambda}$ nor the last component $\left\langle\kappa_{\Gamma} \Gamma \mid \lambda\right\rangle$ of the eigenvectors are affected by the unitary transformation (59). Therefore neither the $S$-matrix nor the phase shifts are affected by the transformation. Nevertheless the wave function is seen from Eq. (60) to be subjected to changes by the transformation; in other words, the off-shell properties of the original and the transformed potentials are different. The non-local phase equivalent transformation generates the ambiguity of the interaction obtained by means of the $J$-matrix version of the inverse scattering theory [70].

The off-shell properties of the two-body interactions play an important role in the formation of the properties of a three-body (and generally many-body) system. Therefore it is interesting to investigate the effect of the non-local phase equivalent transformation on the properties of the ${ }^{6} \mathrm{He}$ nucleus within the three-body cluster model. We note that the non-local phase equivalent transformation preserves the energies of the two-body bound states. However the properties of these states are not preserved, for example, the rms radius 
of the two-body bound system may be affected by the transformation [71]. Hence it is somewhat dangerous to apply the transformation to the $N N$ interaction since it may destroy the description of the deuteron properties. We have already noted that we employ effective $n-n$ interactions that are designed for the effective description of many-body nuclear systems and that are not supposed to be careful in the description of the dinucleon. Nevertheless we suppose that it is more natural to apply the transformation to the $n-\alpha$ interaction since the $n+\alpha$ system does not have a bound state and all the information about this interaction is extracted from the scattering data only. It is clear from the Table 3 (c) that the ${ }^{6} \mathrm{He}$ properties are dominated by the $p$ wave component of the $n-\alpha$ interaction. Therefore we apply the non-local phase equivalent transformation to the $p$ wave component of the $n-\alpha$ potential only.

The non-local phase equivalent transformation for the $n-\alpha$ potential $p$ wave component is constructed in the oscillator basis with $\hbar \omega$ values used in threebody calculations with the respective potential model (see Table 2). The simplest non-local transformation involves a $2 \times 2$ unitary matrix $\left[U_{0}\right]$. Any $2 \times 2$ unitary matrix is known to be a rotation matrix with a single continuous parameter $\gamma$ :

$$
\left[U_{0}\right]=\left[\begin{array}{cc}
\cos \gamma & -\sin \gamma \\
+\sin \gamma & \cos \gamma
\end{array}\right] .
$$

A more complicated non-local transformation involves a $3 \times 3$ rotation matrix $\left[U_{0}\right]$ with two continuous parameters $\gamma$ and $\beta$ :

$$
\left[U_{0}\right]=\left[\begin{array}{ccc}
\cos \beta \cos \gamma & -\cos \beta \sin \gamma & \sin \beta \\
\sin \gamma & \cos \gamma & 0 \\
-\sin \beta \cos \gamma & \sin \beta \sin \gamma & \cos \beta
\end{array}\right] .
$$

Clearly the transformation with the matrix (65) is equivalent to the transformation with the matrix (64) if the Euler angle $\beta=0$.

We present in Fig. 12 the results of variational calculations of the ${ }^{6} \mathrm{He}$ ground and the first excited $0^{+}$states with $\tilde{N}=20$ and of the lowest $1^{-}$state with $\tilde{N}=21$ with various $n-n$ potentials and $n-\alpha$ potentials obtained by applying the phase equivalent transformation (64) to various original potentials. The $\gamma$ dependence of $0^{+}$and $1^{-}$state energies is very interesting. It is seen from Fig. 12 that the simplest non-local phase equivalent transformation of $p$ wave component of the $n-\alpha$ potential only, can completely change the spectrum of the three-body cluster system. The $\gamma$ dependence of the energies is seen to be very similar for all potential models under consideration. The variations of the energies of the excited $0^{+}$and $1^{-}$states are seen to be smaller than the ground state energy variations. There is the $0^{+}$states level crossing at $\gamma \approx-150^{\circ}$ for 

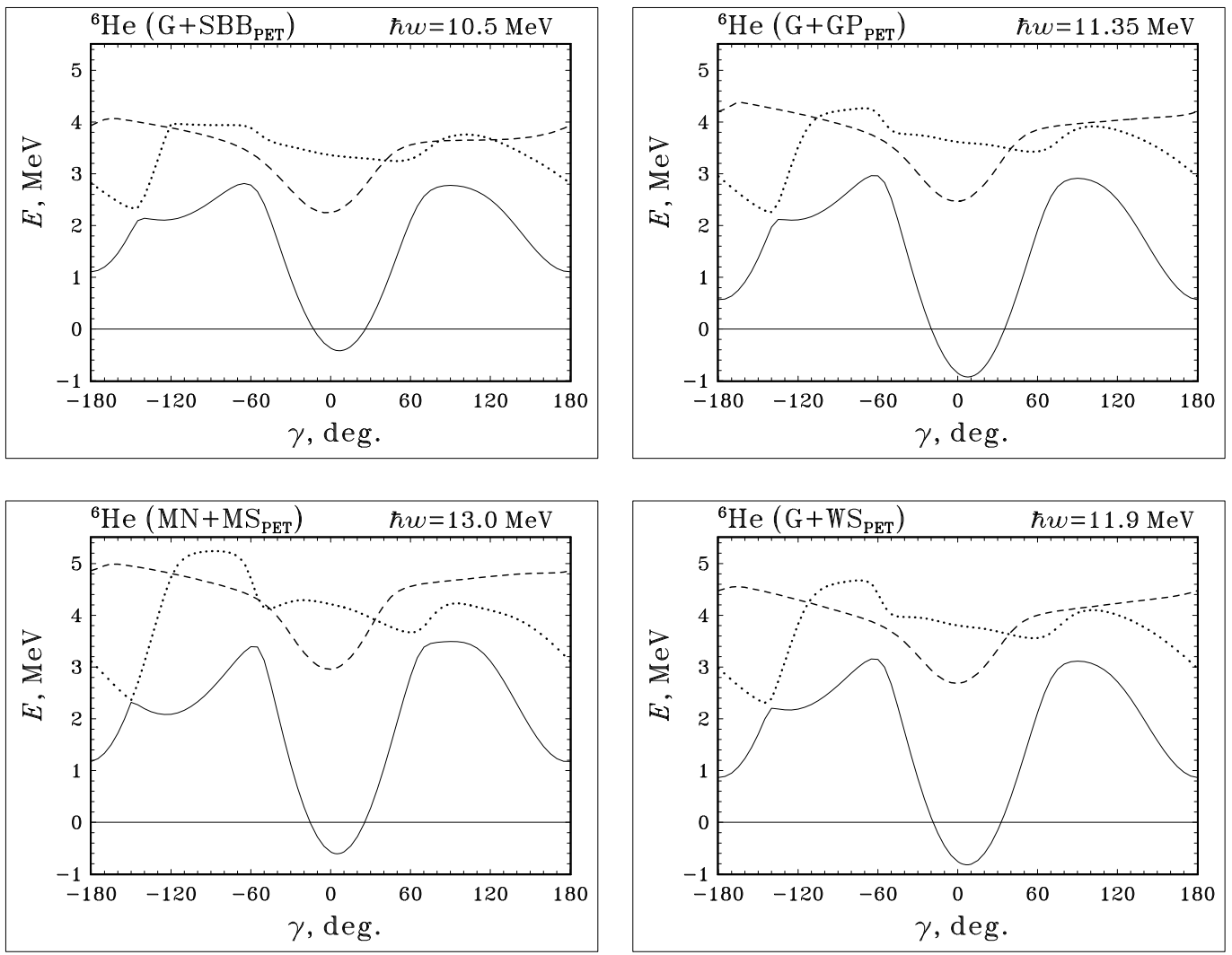

Fig. 12. The ${ }^{6} \mathrm{He}$ ground state energy (solid line) and the energies of the lowest $1^{-}$(dashed line) and $0^{+}$(dotted line) excited states obtained in the variational approach with phase equivalent $n-\alpha$ potentials vs the transformation parameter $\gamma$ of the one-parameter transformation (64). The truncation parameter $\tilde{N}=20$ in the $0^{+}$state calculations and $\tilde{N}=21$ in the $1^{-}$state calculations.

all potential model considered.

The ground state energy $\gamma$ dependence passes through a minimum in the vicinity of $\gamma=5-7^{\circ}$. The minimum corresponds to the increase of the binding by approximately $0.04-0.07 \mathrm{MeV}$ or $7-12 \%$ depending on the potential model, that improves the results obtained with the original potentials $(\gamma=0)$ given in Table 2. The ground states may be additionally shifted down using the twoparameter transformation (65). Supposing $\gamma=7^{\circ}$ and varying the parameter $\beta$ we obtain variational results shown in Fig. 13 . The ground state $\beta$ dependence has a minimum at $\beta \approx-2.5^{\circ}$ for all potential models. This minimum corresponds to the additional binding of $0.019 \div 0.023 \mathrm{MeV}$ (about $2-4 \%$ ).

The variational results presented in Figs. 12 and 13 are interesting for understanding the general trends of variation of the ground state energy and ${ }^{6} \mathrm{He}$ spectrum when the parameters of the phase equivalent transformations (64)(65) are varied in a wide range of values. The corrections to the ${ }^{6} \mathrm{He}$ binding due to the effect of the phase equivalent transformation are better seen in Fig. 14 where we present in a larger scale the $J$-matrix results for the ground 


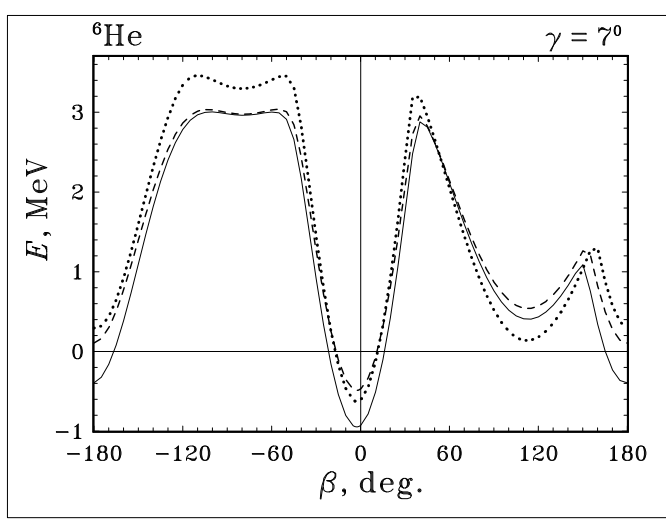

Fig. 13. The ${ }^{6} \mathrm{He}$ ground state energy vs the transformation parameter $\beta$ of the phase equivalent transformation (65) with $\gamma=7^{\circ}$. Solid, dashed and dotted lines are calculations in the variational approximation with $\mathrm{G}+\mathrm{GP}_{\mathrm{PET}}$, $\mathrm{MN}+\mathrm{GP}_{\mathrm{PET}}$ and $\mathrm{MN}+\mathrm{MS}_{\mathrm{PET}}$ potential models, respectively, with $\hbar \omega$ values given in Table 2 and $\tilde{N}=20$.

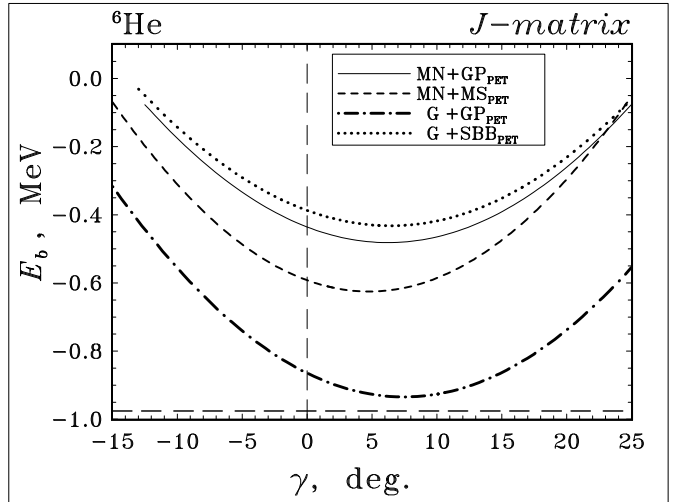

Fig. 14. The ${ }^{6} \mathrm{He}$ ground state energy obtained in the $J$-matrix approach with phase equivalent $n-\alpha$ potentials vs the transformation parameter $\gamma$ of the one-parameter transformation (64). The truncation parameter $\tilde{N}=20$, the $\hbar \omega$ values for each potential model can be found in Table 2. The straight dashed line is the experimental ground state energy.

state energies. The ${ }^{6} \mathrm{He}$ binding energy is seen to be very close to the empirical value in the $\mathrm{G}+\mathrm{GP}_{\mathrm{PET}}$ potential model when $\gamma \approx 7^{\circ}$. However the results presented in Fig. 14 were obtained with not very large value of the truncation parameter $\tilde{N}=20$. With $\tilde{N}=28$ and $\gamma=7.5^{\circ}$ we obtain in this potential model an excellent description of the ${ }^{6} \mathrm{He}$ binding energy $E_{b}=0.952 \mathrm{MeV}$ and rms radius $\left\langle r^{2}\right\rangle^{1 / 2}=2.37 \mathrm{fm}$. Using the two-parameter transformation (65) with $\gamma=7.5^{\circ}$ and $\beta=-3^{\circ}$, we obtain $E_{b}=0.973 \mathrm{MeV}$ and $\left\langle r^{2}\right\rangle^{1 / 2}=2.36 \mathrm{fm}$, i. e. the phenomenological value $E_{b}=0.976 \mathrm{MeV}$ is reproduce nearly exactly.

The correlations between the ${ }^{6} \mathrm{He}$ rms radius $\left\langle r^{2}\right\rangle^{1 / 2}$ and the square root of the binding energy $E_{b}^{1 / 2}$, are depicted in Fig. 15 . We present the $J$-matrix results obtained with various potential models when the parameter $\gamma$ of the phase equivalent transformation (64) varies on the interval where ${ }^{6} \mathrm{He}$ appears to be bound in the variational approximation in the given potential model. The correlations are seen to be very interesting: there are two very different $\left\langle r^{2}\right\rangle^{1 / 2}$ values that are in correspondence with the same binding energy. For $\gamma \lesssim-6^{\circ}$, the rms radius $\left\langle r^{2}\right\rangle^{1 / 2}$ decreases linearly with $E_{b}^{1 / 2}$.

The effect of the phase equivalent transformation (64) on the reduced $E 1$ transition probability $\frac{d \mathcal{B}(E 1)}{d E}$ in ${ }^{6} \mathrm{He}$, is illustrated by Fig. 16 . The effect is seen to be essential. The naive expectation is that the soft dipole mode will be more enhanced if the binding energy is smaller. However the strength of the $E 1$ 


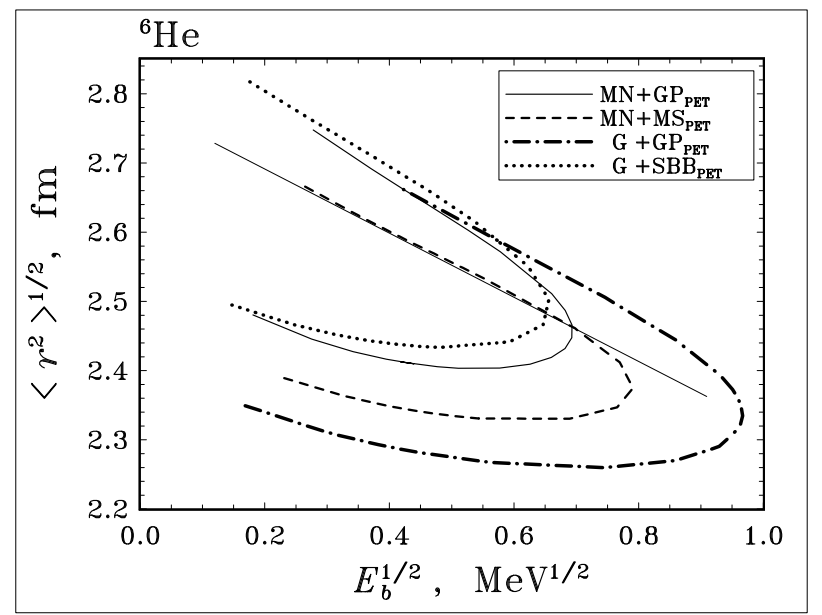

Fig. 15. Correlations between $E_{b}^{1 / 2}$ and rms radius in ${ }^{6} \mathrm{He}$ obtained by variation of the parameter $\gamma$ of the phase equivalent transformation (64) in various potential models ( $J$-matrix approximation with $\tilde{N}=20$, the corresponding $\hbar \omega$ values can be found in Table 2). A straight solid line is added to visualize the linear correlation between $\left\langle r^{2}\right\rangle^{1 / 2}$ and $E_{b}^{1 / 2}$ on a part of the trajectory.

transitions in the vicinity of the maximum of $\frac{d \mathcal{B}(E 1)}{d E}$ does not demonstrate so simple dependence on the binding energy $E_{b}$ (the corresponding $E_{b}$ values are listed in the figure).
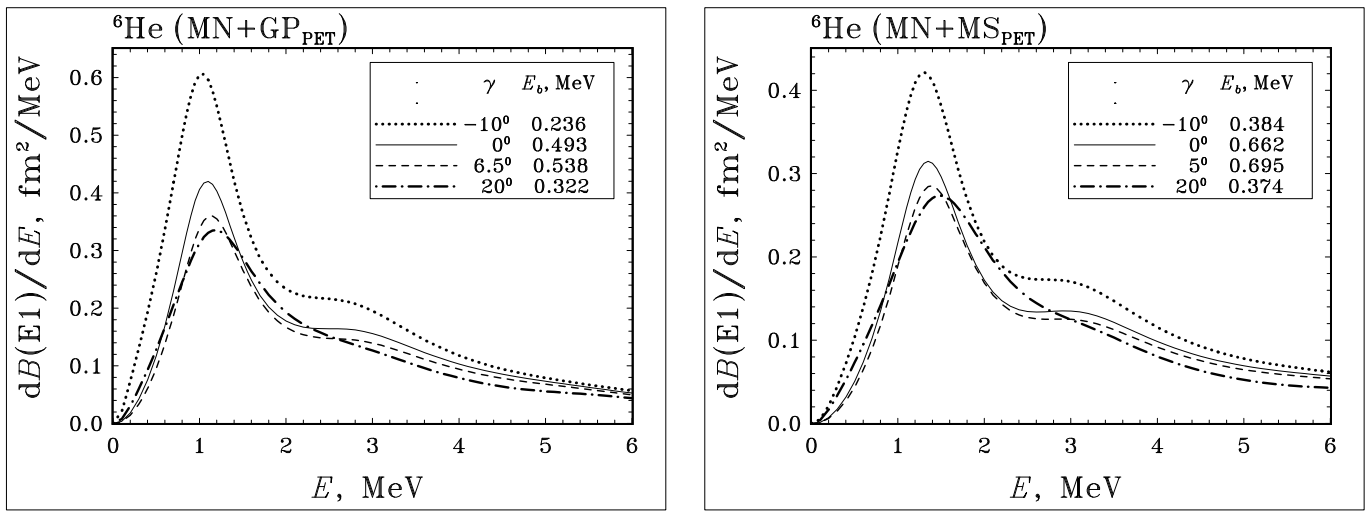

Fig. 16. Reduced $E 1$ transition probability $\frac{d \mathcal{B}(E 1)}{d E}$ in ${ }^{6} \mathrm{He}$ obtained in the JJ approximation with the ground state truncation parameter $\tilde{N}_{\text {g.s. }}=22$ and the final state truncation parameter $\tilde{N}_{\text {f.s. }}=23$ with the MN + GP PET (left panel) and $\mathrm{MN}+\mathrm{MS}_{\mathrm{PET}}$ (right panel) potential models (the corresponding $\hbar \omega$ values can be found in Table 2) and different values of the parameter $\gamma$ of the phase equivalent transformation (64) (the corresponding binding energies are listed in the figure). 


\section{Conclusions}

The hyperspherical $J$-matrix formalism makes it possible to study not only the few-body disintegration of the system but also to calculate the $S$-matrix poles in the $A$-body system. This approach appears to be a very powerful tool for calculations of the bound state properties. As a result, we obtain a unified theory that is capable to investigate in a unique approach both the discrete and continuum spectra of $A$-body systems.

The suggested $J$-matrix motivated non-local phase equivalent transformation may be used to fit binding energies of many-body systems in the case when all information about two-body interaction is extracted from the scattering data only. The transformation can be easily utilized in the studies of many-body systems with any $L^{2}$ basis.

This work was supported in part by the State Program "Russian Universities" and by the Russian Foundation of Basic Research, Grant No 02-02-17316.

\section{References}

[1] E. J. Heller and H. A. Yamani, Phys. Rev. A 9, 1201 (1974); 9, 1209 (1974).

[2] H. A. Yamani and L. Fishman, J. Math. Phys. 16, 410 (1975).

[3] J. T. Broad and W. P. Reinhardt, Phys. Rev. A 14, 2159 (1976); J. Phys. B 9, 1491 (1976).

[4] A. M. Shirokov, Yu. F. Smirnov, and L. Ya. Stotland, in Proc. XIIth Europ. Conf. on Few-Body Phys., Uzhgorod, USSR, 1990 (Ed. V. I. Lengyel and M. I. Haysak), 173 (Uzhgorod, 1990).

[5] Yu. F. Smirnov, L. Ya. Stotland, and A. M. Shirokov, Izv. Akad. Nauk SSSR, Ser. Fiz. 54, No 5, 897 (1990) [Bull. Acad. Sci. USSR, Phys. Ser. 54, No 5, 81 (1990)].

[6] D. A. Konovalov and I. A. McCarthy, J. Phys. B27, L407 (1994); J. Phys. B27, L741 (1994).

[7] G. F. Filippov and I. P. Okhrimenko, Yad. Fiz. 32, 932 (1980) [Sov. J. Nucl. Phys. 32, 480 (1980)]; G. F. Filippov, Yad. Fiz. 33, 928 (1981) [Sov. J. Nucl. Phys. 33, 488 (1981)].

[8] Yu. F. Smirnov and Yu. I. Nechaev, Kinam 4, 445 (1982); Yu. I. Nechaev and Yu. F. Smirnov, Yad. Fiz. 35, 1385 (1982) [Sov. J. Nucl. Phys. 35, 808 (1982)]. 
[9] G. F. Filippov, V. S. Vasilevski, and L. L. Chopovski, Fiz. Elem. Chastits At. Yadra 15, 1338 (1984); 16, 349 (1985) [Sov. J. Part. Nucl. 15, 600 (1984); 16, $153(1985)]$.

[10] G. F. Filippov, Rivista Nuovo Cim. 12, 1 (1989).

[11] V. A. Knyr, A. I. Mazur, and Yu. F. Smirnov, Yad. Fiz. 52, 754 (1990) [Sov. J. Nucl. Phys. 52, 483 (1990)].

[12] V. A. Knyr, A. I. Mazur, and Yu. F. Smirnov, Yad. Fiz. 54, 1518 (1991) [Sov. J. Nucl. Phys. 54, 927 (1991)].

[13] S. P. Merkuriev and L. D. Faddeev, Quantum scattering theory for systems of few bodies (Nauka Publishes, Moscow, 1985).

[14] R. I. Jibuti, Elem. Part. Atom. Nucl. 14, 741 (1983).

[15] R. I. Jibuti and N. B. Krupennikova, Hyperspherical harmonics method in quantum mechanics of few bodies (Metsniereba, Tbilisi, 1984).

[16] M. V. Zhukov, B. V. Danilin, D. V. Fedorov, J. M. Bang, I. J. Thompson, and J. S. Vaagen, Phys. Rep. 231, 151 (1993).

[17] A. M. Shirokov, in Proc. Int. Conf. Perspectives of Nuclear Physics in the Late Nineties, Hanoi, Vietnam, 1994 (Ed. Nguyen Dinh Dang, Da Hsuan Feng, Nguyen Van Giai, Nguyen Dinh Tu), 295 (Singapore, World Scientific, 1995); nucl-th/9407019 (1994).

[18] J. M. Bang, B. V. Danilin, V. D. Efros, J. S. Vaagen, M. V. Zhukov, and I. J. Thompson, Phys. Rep. 264, 27 (1996).

[19] E. Nielsen, D. V. Fedorov, A. S. Jensen, and E. Garrido, Phys. Rep. 347, 373 (2001).

[20] Yu. F. Smirnov and A. M. Shirokov, Preprint ITF-88-47R (Kiev, 1988); A. M. Shirokov, Yu. F. Smirnov, and S. A. Zaytsev, in Modern Problems in Quantum Theory (Ed. V. I. Savrin and O. A. Khrustalev), (Moscow, 1998), 184; Teoret. Mat. Fiz. 117, 227 (1998) [Theor. Math. Phys. 117, 1291 (1998)].

[21] T. Ya. Mikhelashvili, Yu. F. Smirnov, and A. M. Shirokov, Yad. Fiz. 48, 969 (1988) [Sov. J. Nucl. Phys. 48, 617 (1988)]; J. Phys. G 16, 1241 (1990).

[22] D. E. Lanskoy, Yu. A. Lurie, and A. M. Shirokov, Z. Phys. A 357, 95 (1997).

[23] Yu. A. Lurie, Yu. F. Smirnov, and A. M. Shirokov, Izv. Ros. Akad. Nauk, Ser. Fiz. 57, 193 (1993) [Bull. Rus. Acad. Sci., Phys. Ser. 57, 943 (1993)].

[24] A. M. Shirokov, Yu. A. Lurie, and Yu. F. Smirnov, Halo'94 NORDITA/NorFa Study Weekend on Halo Nuclei, Copenhagen, 1994 (Ed. J. M. Bang and J. S. Vaagen).

[25] Yu. F. Smirnov, Yu. A. Lurie, and A. M. Shirokov, Revista Mex. Fis. 40, Supl. 1, 63 (1994). 
[26] Yu. A. Lurie and A. M. Shirokov, Izv. Ros. Akad. Nauk, Ser. Fiz. 61, 2121 (1997) [Bull. Rus. Acad. Sci., Phys. Ser. 61, 1665 (1997)].

[27] I. P. Okhrimenko, Few-body Syst. 2, 169 (1987).

[28] Yu. F. Smirnov and K. V. Shitikova, Fis. Elem. Chast. At. Yad. 8, 847 (1977) [Sov. J. Part. Nucl. 8, 344 (1977)].

[29] J. M. Bang, A. I. Mazur, A. M. Shirokov, Yu. F. Smirnov, and S. A. Zaytsev, Ann. Phys. (NY) 280, 299 (2000).

[30] L. Johansen, A. S. Jensen, and P. G. Hansen, Phys. Lett. B 244, 356 (1990).

[31] W. Benenson, Nucl. Phys. A 588, 11c (1995).

[32] M. Abramowitz and I. A. Stegun (eds.), Handbook on Mathematical Functions (Dover, New York, 1972).

[33] M. V. Zhukov, B. V. Danilin, D. V. Fedorov, J. S. Vaagen, F. A. Gareev, and J. Bang. Phys. Lett. B 265, 19 (1991).

[34] F. Ajzenberg-Selov, Nucl. Phys. A 490, 1 (1988).

[35] I. Tanihata, T. Kobayashi, O. Yamakawa, S. Shimoura, K. Ekuni, K. Sugimoto, N. Takahashi, T. Shimoda, and H. Sato, Phys. Lett. B 206, 592 (1988).

[36] J. S. Al-Khalili, J. A. Tostevin, and I. J. Thompson, Phys. Rev. C 54, 1843 (1996).

[37] J. S. Al-Khalili and J. A. Tostevin, Phys. Rev. Lett. 76, 3903 (1996).

[38] H. Sagawa and M. Houma, Phys. Lett. B 251, 17 (1990); H. Sagawa, N. Takigawa, and N. van Giai, Nucl. Phys. A 543, 575 (1992).

[39] B. V. Danilin, M. V. Zhukov, J. S. Vaagen, I J Thompson, and J. M. Bang, Surrey University Preprint CNP92/17 (1992).

[40] D. Sackett D. et al., Phys. Rev. C 48, 118 (1993); K. Ieki K. et al., Phys. Rev. Lett. 70, 730 (1993); A. Galonsky A. et al., Michigan State University Preprint MSUCL-899 (1993).

[41] A. H. Wapstra, G. Audi, and R. Hoekstra, At. Data Nucl. Data Tables 39, 281 (1988).

[42] P. Navrátil, J. P. Vary, and B. R. Barrett, Phys. Rev. Lett. 84, 5728 (2000); Phys. Rev. C 62, 054311 (2000).

[43] G. E. Brown and A. D. Jackson, The Nucleon-Nucleon Interaction (NorthHolland, Amsterdam, 1976).

[44] B. V. Danilin, M. V. Zhukov, J. S. Vaagen, and J. M. Bang, Phys. Lett. B 302 , 129 (1993).

[45] L. S. Ferreira, E. Maglione, J. M. Bang, I. J. Thompson, B. V. Danilin, M. V. Zhukov, and J. S. Vaagen, Phys. Lett. B 316, 23 (1993). 
[46] T. Kaneko, M. LeMere, and L. C. Tang, University of Minnesota Preprint TPIMINN-91/32-T (1991).

[47] B. V. Danilin, M. V. Zhukov, A. A. Korsheninnikov, and L. V. Chulkov, Yad. Fiz. 53, 71 (1991) [Sov. J. Nucl. Phys. 53, 45 (1991)].

[48] V. I. Kukulin, V. N. Pomerantsev, Kh. D. Razikov, V. T. Voronchev, and G. G. Ryzhikh, Nucl. Phys. A 586, 151 (1995).

[49] J. Bang and C. Gignoux, Nucl. Phys. A 313, 119 (1979).

[50] E. Garrido, D. V. Fedorov, and A. S. Jensen, Nucl. Phys. A 617, 153 (1997).

[51] K. Katō, S. Aoyama, S. Mukai, and I. Ikeda, Nucl. Phys. A 588, 29c (1995); S. Aoyama, S. Mukai, K. Katō, and I. Ikeda, Progr. Theor. Phys. 93, 99 (1995).

[52] E. Hiyama, M. Kamimura, Nucl. Phys. A 588, 35c (1995).

[53] I. Tanihata, D. Hirata, T. Kobayashi, S. Shimoura, K. Sugimoto, and H. Toki, Phys. Lett. B 289, 261 (1992).

[54] I. Tanihata et al., RIKEN Preprint AF-NP-60 (1987).

[55] L. V. Chulkov, B. V. Danilin, V. D. Efros, A. A. Korsheninnikov, and M. V. Zhukov, Europhys. Lett. 8, 245 (1989).

[56] D. Gogny, P. Pires, and R. de Tourreil, Phys. Lett. B 32, 591 (1970).

[57] R. V. Reid, Jr., Ann. Phys. (NY) 50, 411 (1968).

[58] I. J. Thompson, B. V. Danilin, V. D. Efros, J. S. Vaagen, J. M. Bang, and M. V. Zhukov, Phys. Rev. C 61, 024318 (2000).

[59] O. F. Nemets, V. G. Neudatchin, A. E. Rudchik, Yu. F. Smirnov, and Yu. M. Tchuvil'sky, Nucleon associations in atomic nuclei and multi-nucleon transfer reactions (Naukova Dumka Publishers, Kiev, 1988).

[60] M. Moshinsky and Yu. F. Smirnov, The Harmonic Oscillator in Modern Physics (Harwood Academic Publishers, Amsterdam, 1996).

[61] S. Funada, H. Kameyama, and Y. Sakuragi, Nucl. Phys. A 575, 93 (1994).

[62] B. V. Danilin, T. Rogde, S. N. Ershov, H. Heiberg-Andersen, J. S. Vaagen, I. J. Thompson, and M. V. Zhukov, Phys. Rev. C 55, R577 (1997).

[63] R. G. Newton, Scattering theory of waves and particles, 2nd. ed. (SpringerVerlag, New York, 1982).

[64] A. M. Shirokov and V. N. Sidorenko, Yad. Fiz. 63, 2085 (2000) [Phys. At. Nucl. 631993 (2000)].

[65] F. Cooper, A. Khare, and U. Sukhatme, Phys. Rep. 251, 267 (1995).

[66] E. Garrido, D. V. Fedorov, and A. S. Jensen, Nucl. Phys. A 650, 247 (1999). 
[67] F. Coester, S. Cohen, B. Day, and C. W. Vincent, Phys. Rev. C 1, 769 (1970); M. I. Haftel and F. Tabakin, Phys. Rev. C 3, 921 (1971).

[68] H. C. Pradhan, P. U. Sauer, and J. P. Vary, Phys. Rev. C 6, 407 (1972).

[69] A. M. Shirokov, J. P. Vary, T. A. Weber, and A. I. Mazur, Izv. Ros. Akad. Nauk, Ser. Fiz. [Bull. Rus. Acad. Sci., Phys. Ser.] 65, 1572 (2001).

[70] S. A. Zaytsev, Teoret. Mat. Fiz. 115, 263 (1998) [Theor. Math. Phys. 115, 575 (1998)]; 121, 424 (1999) [Theor. Math. Phys. 121, 1617 (1999)].

[71] W. N. Polyzou, Phys. Rev. C 58, 91 (1998). 